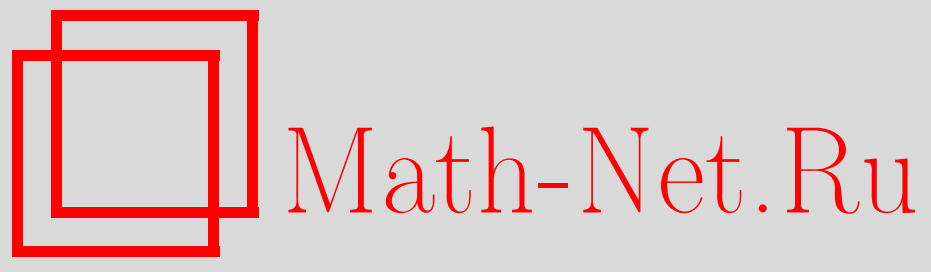

А. К. Гущин, О разрешимости задачи Дирихле для неоднородного эллиптического уравнения второго порядка, Матем. сб., 2015, том 206, номер 10, 71-102

DOI: https://doi.org/10.4213/sm8560

Использование Общероссийского математического портала Math-Net.Ru подразумевает, что вы прочитали и согласны с пользовательским соглашением http://www . mathnet.ru/rus/agreement

Параметры загрузки:

IP: 34.229 .45 .116

26 апреля 2023 г., 09:44:59

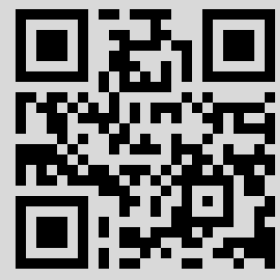




\section{А. К. Гущин \\ О разрешимости задачи Дирихле для неоднородного эллиптического уравнения второго порядка}

Рассматривается обобщающая понятия классического и обобщенного решений постановка задачи Дирихле, в которой решение принадлежит пространству $(n-1)$-мерно непрерывных функций со значениями в пространстве $L_{p}$. Свойство $(n-1)$-мерной непрерывности аналогично классическому определению равномерной непрерывности, но вместо значения функции в точке рассматриваются ее следы на мерах из специального класса - элементы $L_{p}$ по этим мерам. К настоящему времени в рассматриваемой постановке задача исследована недостаточно полно. Прежде всего это относится к условиям на правую часть уравнения, гарантирующим разрешимость задачи. Этому вопросу посвящены основные результаты работы. Обсуждаются термины, в которых такие условия выражаются. Кроме того, исследуется зависимость поведения решения вблизи границы от правой части.

Библиография: 47 названий.

Ключевые слова: эллиптическое уравнение, задача Дирихле, граничное значение.

DOI: $10.4213 / \operatorname{sm} 8560$

\section{§ 1. Введение. Постановка задачи. Основные результаты}

Поставленная еще в 1828 г. К. Гауссом задача Дирихле к настоящему времени исследована весьма детально. Тем не менее настоящая работа посвящена основному вопросу этой теории - условиям ее разрешимости. Поэтому обоснование целесообразности исследований в этом направлении и конкретной постановки изучаемого вопроса требует обстоятельного обсуждения известных результатов в данной области.

1.1. Классическое решение. Прежде всего напомним хорошо известные свойства классического решения, его определение и обсудим условия на коэффициенты уравнения и его правую часть, границу области и граничное значение, при которых такое решение существует. Начнем обсуждение с простейшего случая - уравнения Пуассона. Пусть $D$ - ограниченная область $n$-мерного пространства $\mathbf{R}_{n}$, а $\partial D$ - ее граница. В этой области рассмотрим задачу Дирихле

Исследование выполнено за счет гранта Российского научного фонда (проект № 14-50-00005). 
для уравнения Пуассона

$$
\begin{gathered}
\Delta u(x)=-f(x), \quad x \in D, \\
u(x)=u_{0}(x), \quad x \in \partial D .
\end{gathered}
$$

ОПРедЕЛЕНиЕ 1. Определенная в замыкании $\bar{D}$ области $D$ функция $u$ называется классическим решением задачи Дирихле (1.1), (1.2), если

- она непрерывна в $D$ вместе со своими частными производными до второго порядка включительно $\left(u \in C^{2}(D)\right)$ и для всех точек $x$ этой области значение $\Delta u(x)$ оператора Лапласа от $u$ в точке $x$ совпадает со значением $-f(x)$ в этой точке правой части уравнения $f$,

- она непрерывна в замыкании $\bar{D}$ области $D(u \in C(\bar{D}))$ и для каждой точки границы $\partial D$ значение функции $u$ в этой точке равно значению в этой же точке заданной граничной функции $u_{0}$.

Первые результаты по разрешимости этой задачи для уравнения Лапласа принадлежат П. Дирихле, который, по словам Б. Римана, на своих лекциях (до 1957 г.) доказывал теорему существования решения (принцип Дирихле). Задача сводилась к отысканию функции, на которой интеграл Дирихле $\int_{D}|\nabla v(x)|^{2} d x$ достигает наименьшего значения. Эта глубокая идея получила широкое развитие в теории краевых задач для уравнений с частными производными. Но считать приведенное им рассуждение доказательством существования решения мешает отсутствие четкого описания множества, на котором ищется минимум этого функционала, и обоснования его достижимости.

Доказательство существования решения задачи Дирихле для уравнения Лапласа было получено в 1870 г. К. Нейманом (в случае трех независимых переменных, $n=3$ ) с помощью представления решений потенциалами; утверждение о единственности решения немедленно следует из теоремы о среднем. Метод Неймана давал требуемый результат только в случае выпуклых областей (с дважды гладкой границей) и использовал в то время недоказанное и в общем случае неверное утверждение о существовании и совпадении предельных значений изнутри и извне производных по нормали потенциала двойного слоя. Достаточное для справедливости этого утверждения условие было установлено А. М. Ляпуновым только в 1898 г. в работе [1] (им же были аккуратно доказаны другие, необходимые для применения использованного метода свойства потенциалов простого и двойного слоев). Использование этого результата Ляпунова в доказательстве Неймана привело бы к весьма жестким ограничениям на граничную функцию; не гарантирует выполнение этого свойства даже ее непрерывная дифференцируемость.

Первая попытка распространить метод Неймана на случай невыпуклых областей была предпринята в 1896 г. А. Пуанкаре (см. [2]). Доказательство Пуанкаре использовало предложенный им ранее (см. [3]) специальный метод и, как и доказательство Неймана, основывалось на допущении, что потенциал двойного слоя имеет правильные нормальные производные на $\partial D$. Кроме того, доказательство Пуанкаре опиралось на утверждение (теорема Пуанкаре), в обосновании которого имелся пробел. В 1899 г. А. Корн ликвидировал его 
в случае звездной области $D$ (см. [4]). В том же году В.А. Стеклов в работе [5] (см. также [6]), обосновал справедливость теорем о разрешимости для областей, для которых справедливо обсуждаемое утверждение (теорема Пуанкаре). В 1901 г. С. Заремба доказал справедливость аналогичного теореме Пуанкаре, но более слабого утверждения для любой области, граница которой является поверхностью Ляпунова (см. [7]). Впоследствии, используя этот результат, В.А. Стеклов дал доказательство теоремы о существовании решения задачи Дирихле для уравнения Лапласа в произвольной ограниченной области с ляпуновской границей и получил теорему Пуанкаре как следствие теоремы о разрешимости (подробнее по этому поводу см. [8]). При этом от граничной функции $u_{0}$ требовалась только непрерывность. Таким образом, необходимое для существования решения условие $u_{0} \in C(\partial D)$ оказалось и достаточным.

Не столь изящной является теорема о разрешимости для неоднородного уравнения. Несложно убедиться, что необходимое условие непрерывности правой части не является достаточным. Достаточное для существования решения задачи Дирихле для уравнения Пуассона условие было установлено в 1882 г. О. Гёльдером в его диссертации [9]. Им было введено условие на гладкость функции (сейчас оно называется условием Гёльдера и широко используется в различных областях математики) и было доказано, что если правая часть $f$ удовлетворяет этому условию $\left(f \in C^{\alpha}(D)\right.$ с некоторым положительным показателем $\alpha$ ) и ограничена в $D$, то объемный потенциал с плотностью $f$ является решением в области $D$ уравнения Пуассона (1.1). Условие на $f$ близко к точному. Условие Гёльдера можно ослабить, заменив условием непрерывности по Дини (см., например, монографию [10]). Но отказаться от него нельзя. Кроме того, имеются аргументы, которые делают условие Гёльдера более предпочтительным. При его выполнении, как доказано в 1909 г. А. Корном (см. [11]), производные второго порядка решения задачи Дирихле для уравнения Пуассона также удовлетворяют условию Гёльдера, причем с тем же показателем.

Приведенные результаты распространяются и на уравнения с непрерывными по Гёльдеру переменными коэффициентами; для простоты мы будем говорить об уравнении без младших членов. Теорема о единственности решения задачи Дирихле немедленно следует из принципа максимума; сильный принцип максимума был доказан в 1927 г. в работе Э. Хопфа [12]. Доказательство существования решения базируется на принадлежащей А. Корну (см. [13]) идее замораживания коэффициентов: уравнения с гладкими коэффициентами можно локально рассматривать как возмущения уравнений с постоянными коэффициентами. Проблема существования и основные свойства решений задачи Дирихле были исследованы в 1929-1932 гг. Г. Жиро методом интегральных уравнений, связанным с представлением решения в виде потенциалов (см. [14]-[16]). Другой метод исследования задачи Дирихле был предложен в 1930-х гг. Ю. Шаудером в работах [17] и [18]. Основой этого метода являются априорные оценки решений в $C^{2+\alpha}(\bar{D})$ и разрешимость задачи Дирихле для уравнения Пуассона в этом пространстве. Этим методом было доказано, что для любой правой части из $C^{\alpha}(\bar{D})$ существует решение из $C^{2+\alpha}(\bar{D})$. Таким образом, рассматриваемый дифференциальный оператор осуществляет изоморфизм указанных 
пространств. Возникающее при этом условие $u_{0} \in C^{2+\alpha}(\bar{D})$ не является существенным. От него можно отказаться (конечно, граничная функция должна быть непрерывной на $\partial D$ ), рассматривая весовые (внутренние) гёльдеровы нормы (подробнее по этому поводу см., например, [10]). Непосредственное, не использующее теорему существования доказательство результата о непрерывности по Гёльдеру вторых производных решения (из $C^{2}(D)$ ) было получено в 1932 г. Э. Хопфом в работе [19]. Его метод, основанный на идее Корна возмущения уравнения с постоянными коэффициентами, предвосхищает некоторые важные аспекты теории Шаудера.

Итак, условия на граничную функцию в теоремах о разрешимости задачи Дирихле неулучшаемы. Условия на правую часть близки к точным, они являются неулучшаемыми, если рассматривать решение из $C^{2+\alpha}(\bar{D})$. В такой ситуации неулучшаемым является и условие непрерывности по Гёльдеру коэффициентов уравнения. Естественно возникает вопрос об условиях на гладкость границы. Как показал в 1907 г. А. Лебег (см. [20]), не в любой области существует классическое решение. Насколько можно ослабить условие на границу, при котором В. А. Стекловым была доказана теорема о разрешимости? Нетрудно убедиться, что существенного ослабления этого условия при использовании потенциалов добиться нельзя. Для дальнейшего продвижения в этом направлении необходимы другие идеи и методы. Окончательный ответ на обсуждаемый вопрос дается в терминах решения Перрона и регулярности граничной точки. Предложенный в 1923 г. в работе О. Перрона [21] метод построения решения задачи Дирихле для уравнения Лапласа отделяет задачу существования решения от изучения его граничного поведения. Он элементарен и опирается только на принцип максимума и доказанную еще Г. Шварцем разрешимость задачи Дирихле в шаре. Идея метода принадлежит А. Пуанкаре; И. Г. Петровский называл его методом Пуанкаре-Перрона (см. [22]). Необходимое и достаточное условие регулярности граничной точки, а вместе с ним и условие разрешимости задачи Дирихле, было получено в 1924 г. Н. Винером в работе [23]. С дальнейшим развитием этой тематики можно ознакомиться в работе М. В. Келдыша [24] (см. также [10]). Наличие принципа максимума и разрешимость задачи Дирихле в шаре позволяют распространить метод Перрона на уравнения с переменными коэффициентами и отказаться от условия гладкости границы. Необходимые и достаточные условия на границу области, при которых справедливы теоремы о классической разрешимости, дает доказанная в работе О. А. Олейник [25] теорема об эквивалентности регулярности граничной точки относительно эллиптического оператора и ее регулярности относительно оператора Лапласа. Ссылки на дальнейшие результаты в этом направлении можно найти, например, в [10].

1.2. Обобщенное решение. Введенные С.Л. Соболевым пространства дифференцируемых в обобщенном смысле функций и доказанные им теоремы вложения (см. работы [26]-[29]), оказались мощным аппаратом исследования краевых задач для дифференциальных уравнений. Пространства Соболева идеально приспособлены (см. [27] и [29]) к исследованию смешанных задач и 
задачи Коши для гиперболических уравнений, для которых в классической постановке требования на гладкость начальных функций растут с увеличением числа независимых переменных. Они оказались весьма удобным аппаратом и в теории уравнений эллиптического типа (см. [29]). Расширение класса дифференцируемых функций и ослабление требований на гладкость решения позволило, в частности, с новых позиций взглянуть на задачу Дирихле для линейного эллиптического уравнения второго порядка и получить совсем простое и прозрачное доказательство ее однозначной разрешимости. Именно в таких пространствах удобно рассматривать функционал энергии, на отыскании экстремума которого базируется принцип Дирихле. Отметим, что вариационный подход к задаче Дирихле для уравнения Лапласа в сочетании с методом гильбертова пространства был использован еще в работах Д. Гильберта [30] и А. Лебега [31].

Далее мы ограничимся рассмотрением уравнения в самосопряженной форме без младших членов

$$
\sum_{i, j=1}^{n} \frac{\partial}{\partial x_{i}}\left(a_{i j}(x) \frac{\partial u}{\partial x_{j}}\right)=-f(x)
$$

Здесь $\left(a_{i j}(x)\right), x \in D,-$ заданная симметрическая, равномерно по $x \in D$ положительно определенная матрица с измеримыми и ограниченными элементами:

$$
\gamma \leqslant \sum_{i, j=1}^{n} a_{i j}(x) \xi_{i} \xi_{j} \leqslant \gamma^{-1} \quad \forall \xi=\left(\xi_{1}, \ldots, \xi_{n}\right) \in \mathbf{R}_{n}, \quad|\xi|=1, \quad \text { и п.в. } x \in Q
$$

с некоторой положительной постоянной $\gamma$.

Вариационный подход немедленно приводит к обобщенной постановке задачи Дирихле для уравнения (1.3). Минимум функционала энергии

$$
\int_{D}\left(\sum_{i, j=1}^{n} \frac{\partial v(x)}{\partial x_{i}} a_{i j}(x) \frac{\partial v(x)}{\partial x_{j}}-2 f(x) v(x)\right) d x
$$

ищется на множестве таких функций $v$ из пространства $W_{2}^{1}(D)$, след которых на границе области $D$ совпадает с заданной граничной функцией $u_{0}$; здесь $f \in$ $L_{2}(D)$. Конечно, при этом необходимо требовать, чтобы $u_{0} \in W_{2}^{1 / 2}(\partial D)$. Если на границу не налагать требований, обеспечивающих существование следа, то условие (1.2) следует записывать следующим образом: $v-u_{0} \in \stackrel{\circ}{W}_{2}^{1}(D)$. При этом функцию $u_{0}$ нужно считать заданной на всей области $D$ и принадлежащей пространству $W_{2}^{1}(D)$. Легко видеть, что необходимым условием достижения на функции $u$ экстремума функционала энергии (1.5) является выполнение следующего интегрального тождества

$$
\int_{D}\left(\sum_{i, j=1}^{n} \frac{\partial u}{\partial x_{i}} a_{i j}(x) \frac{\partial \eta}{\partial x_{j}}\right) d x=\langle f, \eta\rangle \quad \text { для всех } \quad \eta \in \stackrel{\circ}{W}_{2}^{1}(D),
$$


здесь $\langle f, \eta\rangle=\int_{D} f \eta d x-$ значение функционала $f$ на элементе $\eta \in \stackrel{\circ}{W}_{2}^{1}(D)$. При этом в качестве $f$ можно брать произвольный линейный ограниченный функционал на пространстве $\stackrel{\circ}{W}_{2}^{1}(D), f \in W_{2}^{-1}(D)$. Так как множество бесконечно дифференцируемых и финитных в $D$ функций плотно в $\stackrel{\circ}{W}_{2}^{1}(D)$, то (1.6) эквивалентно уравнению (1.3), понимаемому как равенство обобщенных функций (см. [32]).

ОПРЕДЕЛЕНИЕ 2. Удовлетворяющая тождеству (1.6) и условию $u-u_{0} \in$ $\stackrel{\circ}{W}_{2}^{1}(D)$ функция $u$ из пространства $W_{2}^{1}(D)$ называется обобщенным решениeм задачи Дирихле для уравнения (1.3) с граничным условием (1.2).

Существование обобщенного решения, его единственность и справедливость оценки решения немедленно следует из теоремы Рисса об общем виде линейного функционала в гильбертовом пространстве. Таким образом, и для обобщенных решений имеет место теорема об изоморфизме.

Итак, теорема об однозначной разрешимости задачи Дирихле для эллиптического уравнения (1.3) справедлива для любой ограниченной области, произвольных $u_{0} \in W_{2}^{1 / 2}(\partial D)$ и $f \in W_{2}^{-1}(D)$. От коэффициентов достаточно требовать только измеримость и ограниченность. Это позволяет переносить некоторые свойства линейных уравнений на квазилинейные (см., например, [33] и [10]).

1.3. Решение из $W_{2, \text { loc }}^{1}$ Простое и удобное в применении понятие обобщенного решения имеет один довольно существенный недостаток. Оно не является в буквальном смысле обобщением понятия классического решения: не любая непрерывная на границе функция является следом функции из пространства $W_{2}^{1}(D)$ (пространство $C(\partial D)$ не содержится в $W_{2}^{1 / 2}(\partial D)$ ). В связи с этим возникает потребность в новом определении решения, в расширении класса граничных функций. Естественным пространством, содержащим $C(\partial D)$ и $W_{2}^{1 / 2}(\partial D)$, является пространство $L_{2}(\partial D)$.

Как было показано в 1960 г. И. Нечасом (см. [34]), оператор, ставящий в соответствие граничной функции $u_{0}$ решение $u$ задачи Дирихле для однородного уравнения и рассматриваемый как оператор из $L_{2}(\partial D)$ в $L_{2}(D)$, является ограниченным оператором. Следовательно, его замыкание определено на всем $L_{2}(\partial D)$. Недостаток такой схемы, прежде всего, заключается в отсутствии явного определения решения. Кроме того, пространство $L_{2}(D)$, в котором находится область значений этого оператора, слишком широкое. Для области с дважды гладкой границей явное определение решения было предложено В. П. Михайловым в работе [35].

ОПредЕлЕНиЕ 3. Принадлежащая пространству $W_{2, \text { loc }}^{1}(D)$ функция $u$ называется решением задачи Дирихле из $W_{2, \mathrm{loc}}^{1}(D)$, если она удовлетворяет тождеству (1.6) для всех бесконечно дифференцируемых и финитных в $D$ функций $\eta$ (является решением уравнения (1.3) в пространстве обобщенных функций $\left.\mathscr{D}^{\prime}(D)\right)$ и граничному условию (1.2) в следующем смысле:

$$
\int_{\partial D}\left(u(x-\delta \nu(x))-u_{0}(x)\right)^{2} d s \rightarrow 0 \quad \text { при } \quad \delta \rightarrow+0 ;
$$


здесь и далее $\nu(x)$ - единичный вектор внешней нормали к $\partial D$ в точке $x \in \partial D$.

Очевидно, что и классическое, и обобщенное решения являются решениями из $W_{2, \text { loc }}^{1}(D)$. Отметим, что для обобщенного решения (из $\left.W_{2}^{1}(D)\right)$ левая часть $(1.7)$ является $o(\delta)$ при $\delta \rightarrow 0$. Такое требование принятия граничного значения с заданным порядком представляется излишним.

При условии гладкости коэффициентов $a_{i j}\left(a_{i j} \in C^{1}(\bar{D})\right)$ в [35] доказано следующее утверждение.

ТЕОрема МихайловА. Для любой $u_{0}$ uз $L_{2}(\partial D)$ и любой $f \in L_{2, \operatorname{loc}}(D)$, удовлетворяющей условию

$$
\int_{D} r^{\theta}(x) f^{2}(x) d x<\infty
$$

в котором $r(x)=\operatorname{dist}(x, \partial D)-$ расстояние от точки $x \in D$ до граници рассматриваемой области, а $\theta<3$, задача Дирихле (1.3), (1.2) однозначно разрешима и для ее решения и справедлива ощенка

$$
\begin{gathered}
\int_{D} r(x)|\nabla u|^{2} d x+\sup _{\delta \in\left(0, \delta_{0}\right)} \int_{\partial D} u^{2}(x-\delta \nu(x)) d s \\
\leqslant \text { const }\left[\left\|u_{0}\right\|_{L_{2}(\partial D)}^{2}+\int_{D} r^{\theta}(x) f^{2}(x) d x\right]
\end{gathered}
$$

с достаточно малым $\delta_{0}>0$.

В степенной шкале условие $\theta<3$ является точным - взять в $(1.8) \theta=3$ нельзя. Для общего уравнения второго порядка (с непрерывными коэффициентами при младших членах) теорема о разрешимости имеет такой же вид, как и для задачи в $W_{2}^{1}(D)$ : собственные значения и соответствующие им собственные подпространства совпадают (см. [35]).

Условие $\partial D \in C^{2}$, обеспечивающее взаимную однозначность (при всех достаточно малых положительных значениях параметра $\delta$ ) отображения $x \leftrightarrow$ $x-\delta \nu(x)$ из (1.7) можно ослабить. Достаточно потребовать, чтобы $\partial D \in C^{1}$ и нормаль $\nu$ удовлетворяла условию Гёльдера (см. [36]) или она была непрерывна по Дини (см. [37]):

$$
|\nu(x)-\nu(y)| \leqslant \omega(|x-y|), \quad x \in \partial Q, \quad y \in \partial Q,
$$

с такой монотонной функцией $\omega$, для которой $\int_{0} \frac{\omega(t)}{t} d t<\infty$. При этом принятие решением своего граничного значения (будем говорить о принятии граничного значения в $L_{p}, p>1$; конечно, при этом и граничная функция $u_{0}$ берется из $L_{p}$ ) следует формулировать в локальных терминах:

для каждой точки $x^{0} \in \partial D$ найдется такая окрестность $V_{x^{0}} \subset \partial D$, что

$$
\int_{V_{x^{0}}}\left(u\left(x-\delta \nu\left(x^{0}\right)\right)-u_{0}(x)\right)^{p} d s \rightarrow 0 \quad \text { при } \quad \delta \rightarrow+0 .
$$

Обсуждать точность условия (1.10) вряд ли имеет смысл. По-видимому, ситуация аналогична случаю классического решения, в котором мы понимали 
бы граничное значение как предел решения по нормали. Для серьезного исследования этого вопроса нужны новые идеи. Но предварительно нужно изменить определение принятия граничного значения; без существования нормали к границе нельзя сформулировать условие (1.11). Далее мы вернемся к этому вопросу.

В работе автора [37] были ослаблены (при $p=2$ ) и условия на коэффициенты уравнения. Достаточно предположить, что они измеримы и ограничены в $D$ и непрерывны по Дини на границе. То есть их значения можно изменить на множестве меры нуль так, что будет выполняться неравенство

$$
\left|a_{i, j}(x)-a_{i, j}(y)\right| \leqslant \omega(|x-y|), \quad i, j=1, \ldots, n, \quad x \in \partial Q, \quad y \in Q ;
$$

не ограничивая общность, функцию $\omega$ можно считать такой же, как и в (1.10). По-видимому, условие (1.12) не является точным. Но совсем отказаться от дополнительного (к измеримости и ограниченности) условия на коэффициенты уравнения нельзя; не будет справедлива теорема о единственности (соответствующий пример приведен в [37]). Ослабление требований на принятие граничного значения приводит к ужесточению условий на коэффициенты на границе. Но каковы точные условия на коэффициенты, не известно. Условия (1.10) и (1.12) мы всюду далее будем считать выполненными.

Обсудим теперь условия на граничную функцию и правую часть уравнения. Но сначала приведем аккуратное определение решения в случае принятия граничного значения в $L_{p}\left(u_{0} \in L_{p}(\partial D)\right)$, которое далее будет в центре нашего внимания.

ОПРЕДЕЛЕНИЕ $3^{\prime}$. Функцию $и$ будем называть решением задачи Дирихле $(1.3),(1.2)$ с граничным значением из $L_{p}$, если она принадлежит пространству $W_{2, \text { loc }}^{1}(D)$, удовлетворяет тождеству (1.6) для всех бесконечно дифференцируемых и финитных в $D$ функций $\eta$ (уравнение (1.3) выполняется в смысле равенства обобщенных функций), ее следы на любой лежащей в $D$ гладкой $(n-1)$-мерной поверхности Г принадлежат $L_{p}(\Gamma)$ и выполнено граничное условие (1.2) в смысле (1.11).

Конечно, условие принадлежности следов $L_{p}$ нужно только при больших значениях $p$. Чтобы избежать этого дополнительного условия естественно было бы в определении решения вместо принадлежности решения пространству $W_{2, \text { loc }}^{1}(D)$ требовать его принадлежность $W_{p, \text { loc }}^{1}(D)$. Но это потребовало бы дополнительных условий на коэффициенты уравнения. При сделанных предположениях в случае $p>2$ решение может не существовать, а для $p \in(1,2)$ не справедливо утверждение о единственности. Не помогает и условие гладкости коэффициентов на границе (см. пример в [38]). Как доказано в этой работе, при сформулированном определении решения задача Дирихле для однородного уравнения однозначно разрешима при сделанных предположениях (1.10) и (1.12) для всех $u_{0}$ из $L_{p}(\partial D)$.

В “гильбертовом случае" $p=2$ исследована разрешимость задачи и для неоднородного уравнения (см. [39]). Так как искомое решение $u \in W_{2, \mathrm{loc}}^{1}(D)$, то 
правая часть уравнения должна представляться в виде

$$
f=g-\operatorname{div} G, \quad G \in\left(L_{2, \operatorname{loc}}(D)\right)^{n}, \quad g \in L_{2, \operatorname{loc}}(D) .
$$

В работе [39] показано (см. замечание 3 в доказательстве леммы 2), что в случае $p=2$ для существования решения достаточно потребовать, чтобы правая часть уравнения $f=g-\operatorname{div} G$ удовлетворяла условиям

$$
\begin{aligned}
& \int_{D} \frac{r(x)}{\omega_{f}(r(x))}|G(x)|^{2} d x<\infty, \\
& \int_{D} \frac{r(x)^{3}}{\left[\omega_{f}(r(x))\right]}|g(x)|^{p} d x<\infty
\end{aligned}
$$

с удовлетворяющей условию Дини функцией $\omega_{f}$.

Целью настоящей работы является исследование разрешимости задачи Дирихле с принятием граничного значения в пространстве $L_{p}$ при $p \neq 2$. При этом мы ограничимся наиболее интересным случаем $1<p<2$. Поскольку случай однородного уравнения и неоднородных граничных условий подробно исследован, то всюду далее мы будем рассматривать задачу с однородным граничным условием

$$
u(x)=0, \quad x \in \partial D .
$$

В изучаемой ситуации для существования решений, конечно, достаточно, как и при $p=2$, выполнения условий (1.13) и (1.14) (из сходимости в $L_{2}$ следует сходимость в $L_{p}$ ). Но такое утверждение будет грубым; ясно, что в этом случае класс правых частей должен быть более широким, ограничения на их рост вблизи границы должны быть более слабыми. Действительно, для существования решения достаточно предположения, что функция $g$ удовлетворяет условию

$$
\int_{D} \frac{r(x)^{2 p-1}}{\left[\omega_{f}(r(x))\right]^{p-1}}|g(x)|^{p} d x<\infty
$$

с функцией $\omega_{f}$, удовлетворяющей условию Дини: $\int_{0} \frac{\omega_{f}(t)}{t} d t<\infty ;$ функцию $\omega_{f}$ будем считать фиксированной. Условие на векторное поле $G$ оставим тем же; поэтому в формулировке теоремы будем считать $G=0$, a $f=g$. Возможность замены условия (1.15) мы обсудим в следующем параграфе при доказательстве теоремы 1 в простейшем случае.

ТеОрема 1. Пусть $1<p<2$ и выполнены условия (1.10), (1.12). Тогда для любой правой части $f$ из $L_{2, \mathrm{loc}}(D)$, удовлетворяющей условию $(1.15)$, существует решение задачи Дирихле (1.3), (1.2 $)$. Это решение единственно и для него справедлива оченка

$$
\begin{aligned}
& \sup _{\xi>0}\left(\frac{1}{\xi} \int_{\{x \in D: r(x)<\xi\}}|u(x)|^{p} d x\right)+\int_{D} r(x)|u(x)|^{p-2}|\nabla u(x)|^{2} d x \\
& \leqslant \operatorname{const} \int_{D} \frac{r(x)^{2 p-1}}{\left[\omega_{f}(r(x))\right]^{p-1}}|f(x)|^{p} d x
\end{aligned}
$$

в которой постоянная зависит толъко от $n, \gamma, \omega, p$ и диаметра области $D$. 
Доказательству теоремы 1 в частном случае посвящен $\S 2$ работы; в общей ситуации она доказана в $\S 4$.

ЗАмечАнИЕ 1. Интересно отметить, что оценка (1.16) позволяет расширить класс правых частей, отказавшись от условия $f \in L_{2, \text { loc }}(D)$. Но это приведет к отказу от требования $u \in W_{2, \text { loc }}^{1}(D)$ в определении решения, без которого не верна теорема единственности. Естественно возникает задача отыскания более широкого класса единственности, содержащего все решения задачи Дирихле для уравнений вида (1.3) с правыми частями из $L_{p, \operatorname{loc}}(D)$, удовлетворяющими условию (1.15). По-видимому, таким условием является принадлежность функции $|u|^{p / 2}$ весовому пространству $W_{2}^{1}$ с весом, равным расстоянию до границы (сходимость второго интеграла в левой части (1.16)). Заметим, что в рассматриваемом случае $p<2$ это условие является более сильным, чем условие принадлежности решения пространству $W_{p}^{1}(D)$, которое не обеспечивает единственность задачи. В случае $p>2$ обсуждаемое условие слабее условия $u \in W_{p}^{1}(D)$, которому решение задачи Дирихле удовлетворять не обязано.

Таким образом, в рассматриваемой постановке задача Дирихле для уравнения (1.3) однозначно разрешима при любой граничной функции из $L_{p}$ и любой правой части из $L_{2, \text { loc }}(D)$, удовлетворяющей условию (1.15). От коэффициентов уравнения достаточно потребовать непрерывность по Дини на границе (условие (1.12)), причем отказаться от этого условия нельзя. Зависимость разрешимости от границы области, как уже отмечалось выше, нуждается в дополнительном исследовании, требует новых идей. Однако предварительно следует изменить постановку рассматриваемой задачи, поскольку в приведенном виде она требует существование нормали. К обсуждению другого определения решения, идейно весьма близкого к понятию классического решения, мы перейдем в следующем пункте.

1.4. ( $n-1)$-мерно непрерывное решение. Основным результатом работы автора [37] является доказательство свойства $(n-1)$-мерной непрерывности решения задачи Дирихле. В [37] было введено понятие $(n-1)$-мерной непрерывности функций и доказан ряд свойств таких функций. В определении $(n-1)$-мерной непрерывности, как и при классическом определении непрерывности, все направления равноправны. Но вместо значений функции в точках следует рассматривать ее следы на борелевских мерах $\mu$ в пространстве $\mathbf{R}_{n}$ с носителем в $\bar{D}$, удовлетворяющих следующему условию:

- существует такая постоянная $C=C(\mu)$, что для всех $x^{0} \in \bar{D}$ и всех $r>0$ выполняется неравенство

$$
\mu\left(B_{x^{0}}(r)\right) \leqslant C r^{n-1}
$$

здесь и далее $B_{x^{0}}(r)$ - шар с центром в точке $x^{0}$ радиуса $r$. Наименьшая из постоянных $C$, с которыми выполнено условие (1.17), называется нормой меры $\mu$; будем обозначать ее через $\|\mu\|$. Заметим, что условие $(1.17)$ близко к определению меры Карлесона, но не совпадает с ним (класс мер Карлесона шире), а $\|\mu\|$ действительно является нормой на пространстве 
зарядов, полная вариация которых удовлетворяет условию (1.17). Это банахово пространство будем обозначать через $\mathscr{M}_{n-1}$.

Множество всех $(n-1)$-мерно непрерывных функций со значениями в $L_{p}$ будем обозначать через $C_{n-1, p}(\bar{D})$. Оно является банаховым пространством с нормой

$$
\|v\|_{C_{n-1}(\bar{D})}^{p}=\sup _{\mu} \frac{1}{\|\mu\|} \int_{\bar{D}}|v(x)|^{p} d \mu,
$$

и множество гладких функций плотно в нем, т.е. $C_{n-1, p}(\bar{D})$ можно рассматривать как пополнение пространства непрерывных в $\bar{D}$ функций с нормой (1.18).

ОПРЕДЕЛЕНИЕ 4. Функция $u$ называется $(n-1)$-мерно непрерывным решением задачи Дирихле $(1.3),(1.2)$ (с $\left.u_{0} \in L_{p}(\partial D)\right)$ или решением из $C_{n-1, p}(\bar{D})$, если

1) $u \in W_{2, \text { lос }}^{1}(D)$ и удовлетворяет (в $\left.\mathscr{D}^{\prime}(D)\right)$ уравнению $(1.3)$,

2) $u \in C_{n-1, p}(\bar{D})$ и ее след на $\partial D$ равен $u_{0}$.

Отметим, что само определение $(n-1)$-мерно непрерывного решения не требует выполнения жестких условий на границу области. Для того чтобы все входящие в него понятия были определены, нужно только существование следа на $\partial D$ функции из $C_{n-1, p}(\bar{D})$. Но и от этого требования можно отказаться. Для этого, как и в случае определения обобщенного решения, следует определить подпространство $\stackrel{\circ}{C-1, p}_{n}(\bar{D})$, являющееся замыканием в $C_{n-1, p}(\bar{D})$ (с нормой $(1.18)$ ) множества непрерывных и финитных в $D$ функций. Граничную функцию $u_{0}$ следует считать заданной во всей области $D\left(u_{0} \in C_{n-1, p}(\bar{D})\right)$ и в определении решения требовать принадлежность $u-u_{0}$ подпространству $\stackrel{\circ}{C}_{n-1, p}(\bar{D})$. Однако разрешимость задачи Дирихле доказана только для случая гладкой границы, предполагается выполнение условия (1.10). Таким образом, ситуация похожа на случай классического решения: если искать решение методом потенциалов, то нужны требования на гладкость границы. Освободиться от них можно, привлекая принципиально другой метод - метод Перрона. По-видимому, и в случае решения из $C_{n-1, p}(\bar{D})$ возможно значительное расширение класса рассматриваемых областей. Этот вопрос требует серьезного изучения.

Обсудим теперь вопрос о разрешимости задачи Дирихле в приведенной постановке (в смысле определения 4). Как и ранее, будем предполагать, что коэффициенты уравнения непрерывны по Дини на границе - удовлетворяют условию (1.12). Без этого условия решение не обязано быть единственным даже в "гильбертовом" случае $p=2$ (соответствующий пример имеется в [37]); не помогает и дополнительное (по сравнению с определением 3) требование на поведение решения вблизи границы. Утверждение о единственности такого решения для $p=2$ было доказано еще в [37], а в общем случае $p>1$ - в [38]. Оно также немедленно следует из доказанного автором в работе [43] значительно более сильного и сложного утверждения, являющегося распространением на рассматриваемую ситуацию теоремы Карлесона (см. [40] и [41]) об $L_{p}$-оценках аналитических функций. Для гармонических функций такая теорема была доказана Л. Хёрмандером (см. [42]). 
Из этого обобщения теоремы Карлесона немедленно следует и существование решения из $C_{n-1, p}(\bar{D})$ для однородного уравнения. Случай неоднородного уравнения более или менее полно был исследован только для $p=2$. В работе [39] доказано, что для существования такого решения достаточно потребовать выполнения условий (1.13) и (1.14) с

$$
\omega_{f}(t)=|\ln t|^{-3 / 2}
$$

(условия (1.10) и (1.12) предполагаются выполненными). Для общего эллиптического уравнения второго порядка (для уравнения с младшими членами) разрешимость задачи Дирихле в обсуждаемой постановке была исследована В. Ж. Думаняном (см. [44] и [45]).

Насколько существенным является такой выбор функции $\omega_{f}(t)$, было неясно. В $\S 3$ показано, что для задачи в шаре условие на правую часть формулируется в других терминах; для простоты там рассматривается случай уравнения Пуассона, но это несущественно.

Теорема 2. Пусть $p \in(1,2), a D=\{x:|x|<1\}-$-диничный шар. Тогда для любой $f \in L_{2, \text { lос }}(D)$, удовлетворяющей условию

$$
\int_{1 / 2}^{1}(1-r)|\ln (1-r)|^{1 /(2 p)}\|f\|_{L_{p}(|x|=r)} d r<\infty
$$

существует решение из $C_{n-1, p}(\bar{D})$ задачи Дирихле (1.1), (1.2 $)$. Это решение единственно, и для него справедлива оценка

$$
\begin{aligned}
& \int_{D} r(x)|u|^{p-2}|\nabla u|^{2} d x+\|u\|_{C_{n-1}(\bar{D})}^{p} \\
& \quad \leqslant \operatorname{const}\left[\left(\int_{1 / 2}^{1}(1-r)|\ln (1-r)|^{1 /(2 p)}\|f\|_{L_{p}(|x|=r)} d r\right)^{p}+\|f\|_{L_{p}(|x|<1 / 2)}^{p}\right] .
\end{aligned}
$$

Условие типа (1.19) требует, чтобы область $D$ допускала "разделение переменных": по группе переменных берется норма в $L_{p}$, а по оставшейся - норма в $L_{1}$ со специальным весом. Оно плохо переносится на случай общей области. Конечно, можно покрыть границу достаточно мелкими областями (например, шарами) и в каждом из них добиться "разделения" переменных, распрямляя границу. Но полученное таким образом условие будет зависеть от выбора покрытия и распрямляющего отображения. Естественный выход из этой ситуации - формулирование условия в терминах весового пространства $L_{p}(D)$. Исследованию разрешимости в $C_{n-1, p}(\bar{D})$ задачи Дирихле для уравнения (1.3) в общей ситуации посвящен $\S 4$.

Теорема 3. Пусть $p \in(1,2)$ и выполнены условия (1.10), (1.12). Тогда для любой $f \in L_{2, \operatorname{loc}}(D)$, удовлетворяющей условию

$$
\int_{D}(r(x))^{2 p-1}|\ln (r(x))|^{(2 p-1) / 2}|f|^{p} d x<\infty,
$$


существует решение задачи Дирихле (1.3), (1.2 $)$. Это решение единственно, и для него справедлива оценка

$$
\begin{aligned}
& \int_{D} r(x)|u|^{p-2}|\nabla u|^{2} d x+\|u\|_{C_{n-1}(\bar{D})}^{p} \\
& \quad \leqslant \operatorname{const} \int_{D}(r(x))^{2 p-1}\left|\ln \frac{r(x)}{R_{0}}\right|^{(2 p-1) / 2}|f|^{p} d x+\|f\|_{L_{p}(\{x \in D: r(x)>1 / 2\})}^{p},
\end{aligned}
$$

в которой постоянная зависит от $n, \gamma, \omega, p$ и диаметра $R_{0}$ области $D$.

Оценка теоремы 3, как и в случае теоремы 1 (см. замечание 1 ), дает возможность установить существование решения без требования принадлежности правой части пространству $L_{2, \text { loc }}(D)$; в общем случае это решение не обязано принадлежать $W_{2, \text { loc }}^{1}(D)$, что не позволяет утверждать его единственность. Вместо этого мы получим принадлежность функции $|u|^{p / 2}$ весовому пространству $W_{2}^{1}$ с весом, равным расстоянию до границы (сходимость первого интеграла в левой части последнего неравенства). И если будет доказано утверждение о единственности из замечания 1 , то оно, очевидно, будет справедливо и в рассматриваемом случае. В $§ 4$ обсуждаются и другие возможные ослабления условия на правую часть.

Чтобы отделить существенную часть доказательств теорем 1 и 3 от технических трудностей, которые преодолеваются с помощью известных методов, эти доказательства разбиваются на две части. Сначала рассматривается задача в шаре; для еще большего упрощения ситуации рассматривается случай уравнения Пуассона, в котором нет необходимости специальным образом сглаживать коэффициенты. Доказательствам в этой простейшей ситуации посвящены $\S 2$ и $\S 3$. В $\S 4$ рассматривается общий случай. При этом основное внимание уделяется возникающим при этом особенностям.

\section{§ 2. Разрешимость задачи Дирихле в шаре}

Пусть область $D$ - единичный шар, $D=\left\{x \in \mathbf{R}_{n}:|x|<1\right\}$, а $u$ - решение задачи Дирихле для уравнения Пуассона (1.1). Напомним, что мы рассматриваем случай $u_{0}=0$, т.е.

$$
u(x)=0, \quad x \in \partial D
$$

2.1. Доказательство существования решения. Предположим сначала, что правая часть рассматриваемого в этом параграфе уравнения Пуассона (1.1) принадлежит пространству $L_{2}(D)$, а ее носитель лежит в $D$. Тогда обобщенное решение этой задачи принадлежит пространству $\stackrel{\circ}{W}_{2}^{1}(D)$. С этим решением мы и будем сейчас работать.

Возьмем произвольные числа $\delta>0, \tau \in(0,1 / 2)$, обозначим $t=1-\tau^{2}$, $\rho=\rho(x, t)=\min \left\{1-|x|^{2}, t\right\}$ и подставим в определяющее решение интегральное тождество пробную функцию $\eta=\rho\left(u^{+}+\delta\right)^{p-2} u^{+}$; здесь и далее $u^{+}=1 / 2(|u|+u)$, аналогично $u^{-}=1 / 2(|u|-u)$. Очевидно, что функция $\eta$ 
принадлежит $\stackrel{\circ}{W}_{2}^{1}(D)$; напомним, что мы рассматриваем случай $p \in(1,2)$. Получим

$$
\begin{gathered}
\iint_{D} f(x) \eta(x) d x=\iint_{D}(\nabla u, \nabla \eta) d x=-2 \iint_{D_{\tau}}\left(u^{+}+\delta\right)^{p-2} u^{+}(\nabla u, x) d x \\
+\iint_{D} \rho\left[(p-2)\left(u^{+}+\delta\right)^{p-3} u^{+}+\left(u^{+}+\delta\right)^{p-2}\right]\left(\nabla u^{+}, \nabla u\right) d x
\end{gathered}
$$

здесь и далее $D_{\tau}=\{x \in D:|x|>\tau\}$ - множество тех точек $D$, в которых $\rho(x, t)=1-x^{2}$.

Второе слагаемое в правой части последнего неравенства преобразуем следующим образом:

$$
\begin{aligned}
\iint_{D} \rho & \frac{(p-2) u^{+}+u^{+}+\delta}{\left(u^{+}+\delta\right)^{3-p}}\left|\nabla u^{+}\right|^{2} d x \\
& =(p-1) \iint_{D} \rho \frac{\left|\nabla u^{+}\right|^{2}}{\left(u^{+}+\delta\right)^{2-p}} d x+(2-p) \delta \iint_{D} \rho \frac{\left|\nabla u^{+}\right|^{2}}{\left(u^{+}+\delta\right)^{3-p}} d x .
\end{aligned}
$$

В силу леммы 2 из работы [38] $|u|^{p / 2} \in \stackrel{\circ}{W}_{2}^{1}(D)$, а следовательно, это слагаемое стремится при $\delta \rightarrow+0$ к

$$
(p-1) \iint_{D} \rho \frac{\left|\nabla u^{+}\right|^{2}}{\left(u^{+}\right)^{2-p}} d x
$$

Так как и подынтегральная функция первого слагаемого в правой части (2.2) оценивается при всех $\delta$ суммируемой функцией

$$
\left|\left(u^{+}+\delta\right)^{p-2} u^{+}(\nabla u, x)\right| \leqslant \frac{2}{p}\left(u^{+}\right)^{p / 2}\left|\nabla\left(u^{+}\right)^{p / 2}\right| \in L_{1}(D),
$$

то, переходя к пределу при $\delta \rightarrow+0$ и интегрируя по частям, имеем

$$
-2 \iint_{D_{\tau}}\left(u^{+}+\delta\right)^{p-2} u^{+}(\nabla u, x) d x \rightarrow \frac{2}{p} \tau \int_{\{|x|=\tau\}}\left(u^{+}\right)^{p} d S+\frac{2 n}{p} \iint_{D_{\tau}}\left(u^{+}\right)^{p} d x .
$$

Очевидно, что и левая часть равенства (2.2) имеет предел. Складывая полученное равенство с таким же равенством для $u^{-}$, получаем оценку

$$
\iint_{D} \rho \frac{|\nabla u|^{2}}{|u|^{2-p}} d x+\int_{\{|x|=\tau\}}|u|^{p} d S+\iint_{D_{\tau}}|u|^{p} d x \leqslant C \iint_{D} \rho|u|^{p-1}|f| d x
$$

здесь и всюду далее через $C=C(n, p)$ будем обозначать постоянные, вообще говоря, различные, зависящие от размерности пространства $n$ и показателя суммирования $p$; в $\S 4$ постоянные будут зависеть еще и от постоянной эллиптичности $\gamma$ из (1.4) и функции $\omega$ из (1.10) и (1.12). Так как правая часть последнего неравенства монотонно не убывает по $t$, то той же величиной оценивается и функция

$$
M(t)=\max _{0 \leqslant \xi \leqslant t} \int_{\left\{1-|x|^{2}=\xi\right\}}|u|^{p} d S
$$


Обозначим еще

$$
J(t)=\iint_{D} \rho \frac{|\nabla u|^{2}}{|u|^{2-p}} d x, \quad I(t)=\iint_{D_{\tau}}|u|^{p} d x, \quad t=1-\tau^{2} .
$$

Тогда установленное неравенство можно записать в виде

$$
J(t)+M(t)+I(t) \leqslant C \iint_{D} \rho|u|^{p-1}|f| d x .
$$

Оценим теперь правую часть неравенства (2.3).

$$
\begin{aligned}
& \iint_{D} \rho|u|^{p-1}|f| d x \leqslant M\left(1-\tau^{2}\right)^{(p-1) / p} \int_{\tau}^{1}\left(1-r^{2}\right)\|f\|_{L_{p}(|x|=r)} d r \\
& \quad+t \int_{1 / 2}^{\tau} M(r)^{(p-1) / p}\|f\|_{L_{p}(|x|=r)} d r+t\|u\|_{L_{p}(\{|x|<1 / 2\})}^{(p-1) / p}\|f\|_{L_{p}(\{|x|<1 / 2\})} .
\end{aligned}
$$

Подставляя эту оценку в (2.3) и используя следующее простое утверждение $a>0, \quad b>0, \quad m>0, \quad 0<\kappa<1, \quad m \leqslant a m^{\kappa}+b \Longrightarrow m \leqslant a^{1 /(1-\kappa)}+\frac{b}{1-\kappa}$, получаем для всех $t \in[0,3 / 4]$ оценку

$$
\begin{aligned}
J(t)+M(t) & +I(t) \leqslant C\left(\int_{\tau}^{1}\left(1-r^{2}\right)\|f\|_{L_{p}(|x|=r)} d r\right)^{p} \\
& +C t \int_{1 / 2}^{\tau} M(r)^{(p-1) / p}\|f\|_{L_{p}(|x|=r)} d r+C t\|f\|_{L_{p}(\{|x|<1 / 2\})}^{p} .
\end{aligned}
$$

В частности, при $t=3 / 4$

$$
\begin{aligned}
& M\left(\frac{3}{4}\right)+J\left(\frac{3}{4}\right)+I\left(\frac{3}{4}\right)=M+J+I \\
& \quad \leqslant C\left[\left(\int_{1 / 2}^{1}\left(1-r^{2}\right)\|f\|_{L_{p}(|x|=r)} d r\right)^{p}+\|f\|_{L_{p}(\{|x|<1 / 2\})}^{p}\right]
\end{aligned}
$$

правую часть последнего неравенства (без постоянной) будем обозначать через $\|f\|_{0}^{p}$. Таким образом, мы доказали следующее утверждение.

ЛЕмма 1. Пусть правая часть $f$ уравнения Пуассона (1.1) принадлежит $L_{2}(D) u \operatorname{supp} f \subset D$. Тогда решение задачи Дирихле (1.1), (2.1) удовлетворяет оченке (2.5) и при всех $t \in[0,3 / 4]$ оценке (2.4).

Основным результатом $\S 2$ является следующая теорема.

Tеорема $1^{\prime}$. Пусть $1<p<2$, a $D=\{x:|x|<1\}-$-диничный шар. Тогда для любой правой части $f$ из $L_{2, \mathrm{loc}}(D)$, удовлетворяющей условию

$$
\|f\|_{0}^{p}=\left(\int_{1 / 2}^{1}\left(1-r^{2}\right)\|f\|_{L_{p}(|x|=r)} d r\right)^{p}+\|f\|_{L_{p}(\{|x|<1 / 2\})}^{p}<\infty,
$$

существует решение задачи Дирихле. Это решение единственно и для него справедлива для всех $t \in[0,3 / 4]$ оценка (2.4). В частности, справедлива и оценка (2.5). 
Отметим, что

$$
\|f\|_{0}^{p} \leqslant \int_{D} \frac{r(x)^{2 p-1}}{\left[\omega_{f}(r(x))\right]^{p-1}}|f(x)|^{p} d x,
$$

а следовательно, утверждение теоремы 1 для задачи Дирихле для уравнения Пуассона в шаре немедленно следует из теоремы $1^{\prime}$.

ДОКАЗАТЕЛЬСТво ТЕОРЕмЫ $1^{\prime}$. Возьмем произвольную функцию $f$ из $L_{2, \text { lос }}(D)$, удовлетворяющую условию (2.6). Обозначим через $f_{k}$ функцию, значения которой равны $f$ при $|x|<1-1 / k$ и равны нулю в остальных точках шара. Пусть $u_{k} \in \stackrel{\circ}{W}_{2}^{1}(D)$ - решения задачи Дирихле для уравнения Пуассона с правой частью $f_{k}$. Тогда в силу оценки $(2.5)$ леммы 1 последовательность $\left\{u_{k}\right\}$ фундаментальна в $L_{p}(D)$; обозначим через $u$ ее предел.

Во-первых, так как для любой подобласти $D^{\prime}$, компактно принадлежащей $D$, функция $u_{k}-u_{m}$ при достаточно больших $k$ и $m$ является решением однородного уравнения в некоторой окрестности $\overline{D^{\prime}}$, то по лемме 1 из работы [38] последовательность $\left\{u_{k}\right\}$ сходится и в $W_{2}^{1}\left(D^{\prime}\right)$. Таким образом, $u \in W_{2, \mathrm{loc}}^{1}(D)$.

В рассматриваемой ситуации ссылка на лемму 1 из работы [38], конечно, является излишней (это замечание относится и к некоторым другим моментам доказательства); эта ссылка нужна в случае общего уравнения (1.3). Мы приводим доказательство, перенесение которого на общую ситуацию требует минимальных изменений.

Далее. Из сходимости $\left\{u_{k}\right\}$ к $u$ в $W_{2}^{1}$ на любом лежащем в $D$ компакте следует, что предел $u$ является решением уравнения в $D$. Выполнение граничного условия (2.1) вытекает из стремления к нулю при $k \rightarrow \infty$ и $m \rightarrow \infty$ последовательности значений функционалов $M$, построенных по $u_{k}-u_{m}$. Справедливость оценки (2.4) очевидна: значения функционалов $M$ и $I$ являются пределами соответствующих величин для $u_{k}$, а оценка $J$ следует из теоремы Фату. Отметим, что из несложно проверяемой оценки

$$
\begin{aligned}
& \int_{D} r(x)|\nabla u(x)|^{p} d x \\
& \quad \leqslant\left(\int_{D} r(x)|u(x)|^{p-2}|\nabla u(x)|^{2} d x\right)^{p / 2}\left(\int_{D} r(x)|u(x)|^{p} d x\right)^{(2-p) / 2}
\end{aligned}
$$

следует сходимость последовательности $\left\{u_{k}\right\}$ к $u$ в $W_{p}^{1}(D)$, поэтому производные (по некоторой последовательности) сходятся п.в. (по некоторой подпоследовательности) к соответствующим производным $u$. Теорема доказана.

Как отмечалось в замечании 1 , оценка (2.5) позволяет отказаться от требования принадлежности правой части $L_{2, \text { loc }}(D)$. Конечно, при этом нельзя утверждать, что предел решений с приближающими $f$ правыми частями принадлежит пространству $W_{2, \text { loc }}^{1}(D)$. А поэтому нельзя утверждать и единственность решения. То, что предел решений будет решением предельного уравнения, следует из вытекающей из (2.7) сходимости решений в пространстве $W_{p}^{1}(D ; r(x))$ (в пространстве $W_{p}^{1}$ с весом $r(x)$.

ЗАМЕЧАНИЕ 2. Можно ослаблять и условие на гладкость правой части уравнения. Во-первых, вблизи границы (при $|x|>1 / 2$ ) можно считать $f=g \mu$, где 
$g$ является непрерывной функцией со значениями в $L_{p}$ на единичной сфере $S_{1}$, а $\mu$ - борелевская мера на $\mathbf{R}_{1}$ (с носителем на $[1 / 2,1]$ ). Можно в качестве $f$ брать такую обобщенную функцию, что

$$
\left|\left\langle f, \rho|v|^{p-1}\right\rangle\right| \leqslant \operatorname{const}\left(\|v\|_{W_{p}^{1}(D ; r(x))}+\|v\|_{C\left([1 / 2,1] \rightarrow L_{p}\right)\left(S_{1}\right)}\right)^{p-1} .
$$

2.2. Поведение решения вблизи границы. Прежде всего отметим, что в силу (2.4) для любой удовлетворяющей условию (2.6) функции $f$ из $L_{2, \text { loc }}(D)$ величины $J(t), M(t)$ и $I(t)$ стремятся к нулю при $t \rightarrow 0(\tau \rightarrow 1)$. В п. 2.2 мы покажем, что порядок этого убывания несложно зависит от порядка роста $f$ вблизи границы.

Пусть монотонно неубывающая функция $\omega_{f}(t), t \in[0,3 / 4]$, стремится к нулю при $t \rightarrow 0$ и функция $t / \omega_{f}^{p}(t)$ монотонно не убывает. Потребуем, чтобы правая часть уравнения Пуассона удовлетворяла более жесткому, чем (2.6), условию

$$
\|f\|_{\omega}^{p}=\left(\int_{1 / 2}^{1} \frac{\left(1-r^{2}\right)}{\omega_{f}\left(1-r^{2}\right)}\|f\|_{L_{p}\left(S_{r}\right)} d r\right)^{p}+\frac{3}{4 \omega_{f}^{p}(3 / 4)}\|f\|_{L_{p}(\{|x|<1 / 2\})}^{p}<\infty,
$$

где $S_{r}=\left\{x \in \mathbf{R}_{n}:|x|=r\right\}$ - сфера радиуса $r$; числовой множитель в последнем слагаемом взят для удобства. Подставим в правую часть неравенства (2.4) оценку

$$
M(t) \leqslant M \leqslant C\left[\left(\int_{1 / 2}^{1}\left(1-r^{2}\right)\|f\|_{L_{p}(|x|=r)} d r\right)^{p}+\|f\|_{L_{p}(\{|x|<1 / 2\})}^{p}\right], \quad 0 \leqslant t \leqslant \frac{3}{4},
$$

и воспользуемся монотонностью функций $\omega_{f}(t)$ и $t / \omega_{f}^{p}(t)$. Получим

$$
\begin{aligned}
J(t)+ & M(t)+I(t) \leqslant C\left(\int_{\tau}^{1}\left(1-r^{2}\right)\|f\|_{L_{p}(|x|=r)} d r\right)^{p} \\
& +C t M^{(p-1) / p} \int_{1 / 2}^{\tau}\|f\|_{L_{p}(|x|=r)} d r+C t\|f\|_{L_{p}(\{|x|<1 / 2\})}^{p} \\
\leqslant & C\left[\omega_{f}^{p}(t)\left(\|f\|_{\tau}^{\prime}\right)^{p}+\omega_{f}(t) M^{\kappa}\|f\|_{\tau}^{\prime \prime}+\omega_{f}^{p}(t) \frac{3}{4 \omega_{f}^{p}(3 / 4)}\|f\|_{L_{p}(\{|x|<1 / 2\})}^{p}\right]
\end{aligned}
$$

здесь и далее в этом пункте

$$
\begin{gathered}
\kappa=\frac{p-1}{p}, \quad 0<\kappa<1, \quad\|f\|_{\tau}^{\prime}=\int_{\tau}^{1} \frac{\left(1-r^{2}\right)}{\omega_{f}\left(1-r^{2}\right)}\|f\|_{L_{p}(|x|=r)} d r \\
\|f\|_{\tau}^{\prime \prime}=\int_{1 / 2}^{\tau} \frac{1-r^{2}}{\omega_{f}\left(1-r^{2}\right)}\|f\|_{L_{p}(|x|=r)} d r
\end{gathered}
$$

Подставляя полученную таким образом оценку $M(t)$ опять в $(2.4)$, имеем

$$
\begin{aligned}
J(t)+M(t)+I(t) \leqslant C & \omega_{f}^{p}(t)\left(\|f\|_{\tau}^{\prime}\right)^{p} \\
& +C t \int_{1 / 2}^{\tau} C^{\kappa}\left[\left(\omega_{f}\left(1-r^{2}\right)\|f\|_{r}^{\prime}\right)^{p}+\omega_{f}\left(1-r^{2}\right) M^{\kappa}\|f\|_{r}^{\prime \prime}\right.
\end{aligned}
$$




$$
\begin{aligned}
& \left.\left.\quad+\omega_{f}^{p}\left(1-r^{2}\right) \frac{3}{4 \omega_{f}^{p}(3 / 4)}\|f\|_{L_{p}(\{|x|<1 / 2\})}^{p}\right)\right]^{\kappa}\|f\|_{L_{p}(|x|=r)} d r \\
& +C \omega_{f}^{p}(t) \frac{3}{4 \omega_{f}^{p}(3 / 4)}\|f\|_{L_{p}(\{|x|<1 / 2\})}^{p} \\
& \leqslant C^{1+\kappa}\left(2 \omega_{f}^{p}(t)\|f\|_{\omega}^{p}+M^{\kappa^{2}} \omega_{f}^{1+\kappa}(t)\|f\|_{\omega}^{1+\kappa}\right) .
\end{aligned}
$$

Продолжая этот процесс, на $k$-м шаге получим

$$
\begin{aligned}
J(t) & +M(t)+I(t) \\
& \leqslant C^{1+\kappa+\cdots+\kappa^{k-1}}\left(2 \omega_{f}^{p}(t)\|f\|_{\omega}^{p}+M^{\kappa^{k}} \omega_{f}^{1+\kappa+\cdots+\kappa^{k-1}}\|f\|_{\omega}^{1+\kappa+\cdots+\kappa^{k-1}}\right) .
\end{aligned}
$$

Откуда

$$
J(t)+M(t)+I(t) \leqslant 3 C^{p} \omega_{f}^{p}(t)\|f\|_{\omega}^{p} .
$$

Таким образом, доказано следующее утверждение.

Теорема 4. Пусть правая часть уравнения Пуассона (1.1) удовлетворяет условию (2.8). Тогда для решения и задачи Дирихле (1.1), (2.1) справедлива оченка (2.9).

СлЕДСТвИЕ. При выполнении условия (2.8) справедливо равенство

$$
J(t)+M(t)+I(t)=o\left(\omega_{f}^{p}(t)\right) \quad \text { npu } \quad t \rightarrow 0 .
$$

ДокАЗАТЕЛЬСтво. Подставляя (2.9) в (2.4), получаем

$$
\begin{aligned}
& \frac{J(t)+M(t)+I(t)}{\omega_{f}(t)} \leqslant \operatorname{const}\left(\int_{\tau}^{1} \frac{\left(1-r^{2}\right)}{\omega_{f}\left(1-r^{2}\right)}\|f\|_{L_{p}(|x|=r)} d r\right)^{p} \\
& + \text { const } \frac{t}{\omega_{f}(t)} \int_{1 / 2}^{\tau}\|f\|_{\omega}^{p-1} \omega_{f}^{p-1}\left(1-r^{2}\right)\|f\|_{L_{p}(|x|=r)} d r+\text { const } t\|f\|_{L_{p}(\{|x|<1 / 2\})}^{p}
\end{aligned}
$$

Сформулированное утверждение теперь следует из теоремы Лебега.

\section{§ 3. $(n-1)$-мерная непрерывность решения задачи Дирихле в шаре}

В п. 3.1 приведем определение и некоторые свойства $(n-1)$-мерно непрерывных функций. А в п. 3.2 перейдем к исследованию разрешимости задачи Дирихле в пространстве таких функций.

3.1. Пространство $C_{n-1, p}(\bar{D})$. Рассмотрим неотрицательные борелевские меры $\mu$ в $\mathbf{R}_{n}$ с носителем в $\bar{D}$, удовлетворяющие следующему условию:

существует такая постоянная $C=C(\mu)$, что для всех $x^{0} \in \bar{D}$ и всех $r>0$ выполняется неравенство (1.17)

$$
\mu\left(B_{x^{0}}(r)\right) \leqslant C r^{n-1}
$$

$B_{x^{0}}(r)$ - шар с центром в точке $x^{0}$ радиуса $r$. 
Наименьшую из постоянных $C$, с которыми выполнено условие (1.17), будем называть нормой меры $\mu$ и обозначать $\|\mu\|$. Заметим, что $\|\mu\|$ действительно является нормой на пространстве зарядов, полная вариация которых удовлетворяет условию (1.17). Это банахово пространство будем обозначать через $\mathscr{M}_{n-1}$.

Приведем еще одно, эквивалентное приведенному во введении, определение $(n-1)$-мерной непрерывности. Пусть $\mu$ и $v$ - удовлетворяющие (1.17) единичные меры $(\|\mu\|<\infty,\|v\|<\infty)$. И пусть $\phi-$ мера в $\mathbf{R}_{2 n}$, проекция которой на пространство первых $n$ переменных совпадает с $\mu$, а на пространство остальных $n$ переменных совпадает с $v$ :

$$
\phi\left(G \times \mathbf{R}_{n}\right)=\mu(G), \quad \phi\left(\mathbf{R}_{n} \times G\right)=v(G)
$$

для всех борелевских множеств $G \subset \mathbf{R}_{n}$; будем говорить, что $\phi$ соединяет меры $\mu$ и $v$.

Зафиксируем некоторое число $p>1$. Функцию $v$, принадлежащую пространствам $L_{p}(\bar{D} ; \mu)$ для всех мер $\mu$ из $\mathscr{M}_{n-1}$, будем называть $(n-1)$-мерно непрерывной функиией со значениями в $L_{p}$ (или просто $(n-1)$-мерно непрерывной функцией), если для любого $\varepsilon>0$ существует такое $\delta>0$, что для всех мер $\mu$ и $v$ из рассматриваемого класса и любой соединяющей их меры $\phi$, удовлетворяющих условию

$$
\iint_{\mathbf{R}_{2 n}}|x-y| d \phi(x, y)<\delta
$$

(расстояние между мерами $\mu$ и $v$ вдоль $\phi$ меньше $\delta$ ), выполняется оценка

$$
\frac{1}{\|\mu\|+\|v\|} \iint_{\bar{D} \times \bar{D}}|v(x)-v(y)|^{p} d \phi(x, y)<\varepsilon
$$

(значения функции $v$ на $\mu$ и $v$ вдоль $\phi$ отличаются меньше, чем на $\varepsilon^{1 / p}$ ). Множество всех $(n-1)$-мерно непрерывных функций со значениями в $L_{p}$ будем обозначать через $C_{n-1, p}(\bar{D})$. Оно является банаховым пространством с нормой (1.18)

$$
\|v\|_{C_{n-1, p}(\bar{D})}=\left(\sup _{\mu} \frac{1}{\|\mu\|} \int_{\bar{D}}|v(x)|^{p} d \mu\right)^{1 / p}
$$

и множество гладких функций плотно в нем, т.е. $C_{n-1, p}(\bar{D})$ является пополнением пространства непрерывных в $\bar{D}$ функций с нормой (1.18). Понятие $(n-1)$-мерной непрерывности функций было введено в работе автора [37]. Там же приведено еще одно определение $(n-1)$-мерной непрерывности, доказана эквивалентность этих определений и установлены свойства таких функций. Сразу из определения нормы (1.18) видно, что $(n-1)$-мерно непрерывные функции естественно рассматривать как отображение, ставящее в соответствие каждой мере из $\mathscr{M}_{n-1}$ функцию из пространства $L_{p}$ по этой мере; значение этого отображения на мере будем называть следом на этой мере (или на ее носителе; можно определить след на любом множестве положительной $(n-1)$-мерной меры Хаусдорфа). Множество следов всех функций из $C_{n-1, p}(\bar{D})$ на гладкой 
$(n-1)$-мерной поверхности $\Gamma$ (на мере Лебега на этой поверхности) совпадает c $L_{p}(\Gamma)$.

Установим еще одно свойство функций из $C_{n-1, p}(\bar{D})$, которое нам понадобится в $\S 4$.

Лемма 2. Пусть набор открытых множеств $\left\{D_{0}, D_{1}, \ldots, D_{N}\right\}$ образует покрытие замыкания области D,

$$
\bar{D} \subset \bigcup_{0 \leqslant k \leqslant N} D_{k}, \quad Q_{k}=D_{k} \cap D,
$$

и пусть функиия и принадлежит $C_{n-1, p}\left(\overline{Q_{k}}\right)$ для всех $k=0,1, \ldots, N$. Тогда $u \in C_{n-1, p}(\bar{D})$ и справедлива оценка

$$
\|u\|_{C_{n-1, p}(\bar{D})} \leqslant \sum_{k=0}^{N}\|u\|_{C_{n-1, p}\left(\overline{Q_{k}}\right)} .
$$

ДокАЗАтельство. Пусть $\zeta_{0}, \zeta_{1}, \ldots, \zeta_{N}$ - соответствующее рассматриваемому покрытию разложение единицы. Для каждого $k$ возьмем последовательность $\left\{v_{k, m}\right\}$ непрерывных функций, сходящуюся в $C_{n-1}\left(\overline{Q_{k}}\right)$ к $u$. Тогда последовательность функций $\zeta_{k} v_{k, m}$, продолженных нулем вне $\overline{Q_{k}}$, сходится к $\zeta_{k} u$ в $C_{n-1, p}(\bar{D})$. Следовательно, и функция $u=\bigcup \zeta_{k} u$ принадлежит пространству $C_{n-1, p}(\bar{D})$. Утверждаемая оценка немедленно следует из неравенства треугольника.

Лемма 3. Пусть $v \in C_{n-1, p}(\bar{D})$. Тогда для любого $\alpha \in(0,1)$ функиия $|v|^{\alpha}$ принадлежит $C_{n-1, p / \alpha}(\bar{D})$. При этом

$$
\|v\|_{C_{n-1, p}(\bar{D})}=\left\||v|^{\alpha}\right\|_{C_{n-1, p / \alpha}(\bar{D})} .
$$

ДокАЗАТЕльство. Пусть последовательность непрерывных функций $\left\{v_{k}\right\}$ сходится в $C_{n-1, p}(\bar{D})$ к функции $v$. В силу очевидного неравенства

$$
\left.|| u\right|^{\alpha}-|v|^{\alpha}|\leqslant| u-\left.v\right|^{\alpha}
$$

для любой меры $\mu \in \mathscr{M}_{n-1}$ справедлива оценка

$$
\left.\frac{1}{\|\mu\|} \int_{D}|| v\right|^{\alpha}-\left.\left|v_{k}\right|^{\alpha}\right|^{p / \alpha} d \mu \leqslant \frac{1}{\|\mu\|} \int_{D}|| v|-| v_{k}||^{p} d \mu .
$$

Следовательно, последовательность непрерывных функций $\left\{\left|v_{k}\right|^{\alpha}\right\}$ сходится к $|u|^{\alpha}$. Справедливость равенства норм очевидна.

3.2. Доказательство теоремы 2. Рассмотрим, как и в $\S 2$, сначала случай $f \in L_{2}(D), \operatorname{supp} f \subset D, u$ - решение задачи Дирихле $(1.1),(2.1) ; u \in \stackrel{\circ}{W}_{2}^{1}(D)$. Возьмем произвольное число $\delta>0, \rho(t)=t\left(-\ln \frac{t}{2}\right)^{1 / 2}, t=1-\tau^{2}$, и подставим в интегральное тождество пробную функцию

$$
\eta_{\delta}(x)=\rho\left(1-|x|^{2}\right)\left(u^{+}(x)+\delta\right)^{p-2} u^{+}(x) \in \stackrel{\circ}{W}_{2}^{1}(D) .
$$


Получим

$$
\begin{aligned}
\iint_{D} f \eta_{\delta} d x & \\
= & \iint_{D}\left(u^{+}+\delta\right)^{p-2} u^{+}\left(-2\left(-\ln \frac{1-|x|^{2}}{2}\right)^{1 / 2}+\frac{1}{\left(-\ln \frac{1-|x|^{2}}{2}\right)^{1 / 2}}\right)(x, \nabla u) d x \\
& \quad+\iint_{D} \rho\left(1-|x|^{2}\right) \frac{(p-1) u^{+}+\delta}{\left(u^{+}+\delta\right)^{3-p}}\left|\nabla u^{+}\right|^{2} d x \\
\geqslant & \iint_{D}\left(-2\left(-\ln \frac{1-|x|^{2}}{2}\right)^{1 / 2}+\frac{1}{\left(-\ln \frac{1-|x|^{2}}{2}\right)^{1 / 2}}\right)\left(u^{+}+\delta\right)^{p-2} u^{+}|x| \frac{\partial\left(u^{+}\right)}{\partial r} d x \\
& \quad+(p-1) \iint_{D} \rho\left(1-|x|^{2}\right) \frac{\left|\nabla u^{+}\right|^{2}}{\left(u^{+}+\delta\right)^{2-p}} d x
\end{aligned}
$$

здесь $r=|x|$ - сферическая координата точки. Складывая полученное неравенство с аналогичным неравенством для $u^{-}$(при этом подынтегральные функции отличны от нуля на непересекающихся множествах $\{u(x)>0\}$ и $\{u(x)<0\})$ и устремляя $\delta$ к нулю, получаем неравенство

$$
\begin{aligned}
& \iint_{D} \rho\left(1-|x|^{2}\right) \frac{|\nabla u(x)|^{2}}{u^{2-p}(x)} d x+\iint_{D}\left(-2\left(-\ln \frac{1-|x|^{2}}{2}\right)^{1 / 2}+\frac{1}{\left(-\ln \frac{1-|x|^{2}}{2}\right)^{1 / 2}}\right) \\
& \quad \times|x| \frac{\partial\left(|u(x)|^{p}\right)}{\partial r} d x \leqslant C \iint_{D}|f| \eta d x
\end{aligned}
$$

Преобразуем второе слагаемое из левой части (3.1). В силу принадлежности решения пространству $\stackrel{\circ}{W}_{2}^{1}(D)$ (или в силу следствия из теоремы 4 с $\omega_{f}(t)=$ $\left.(-\ln t+\ln 2)^{-1 /(2 p)}\right)$ при интегрировании этого члена по частям интеграл по границе будет равен нулю. Оно имеет вид

$$
\begin{gathered}
\iint_{D}|u|^{p}\left(2 n\left(-\ln \frac{1-|x|^{2}}{2}\right)^{1 / 2}+\frac{2|x|^{2}}{\left(1-|x|^{2}\right)\left(-\ln \frac{1-|x|^{2}}{2}\right)^{1 / 2}}\right. \\
\left.+\frac{|x|^{2}}{\left(1-|x|^{2}\right)\left(-\ln \frac{1-|x|^{2}}{2}\right)^{3 / 2}}\right) d x-\iint_{D}|u|^{p} \frac{n}{\left(-\ln \frac{1-|x|^{2}}{2}\right)^{1 / 2}} d x .
\end{gathered}
$$

Откуда

$$
\begin{gathered}
\iint_{D} \rho\left(1-|x|^{2}\right) \frac{|\nabla u(x)|^{2}}{u^{2-p}(x)} d x+\iint_{D}|u|^{p} \frac{2|x|^{2}}{\left(1-|x|^{2}\right)\left(-\ln \frac{1-|x|^{2}}{2}\right)^{1 / 2}} d x \\
\leqslant C\left(\|u\|_{L_{p}(D)}^{p}+\iint_{D}|f(x)| \rho\left(1-|x|^{2}\right)|u|^{p-1} d x\right) .
\end{gathered}
$$

Поскольку из $f \in L_{2, \operatorname{loc}}(D)$ и (1.19) следует выполнение условий теоремы $1^{\prime}$, то первое слагаемое в правой части (3.2) оценивается через $\|f\|_{0}^{p}$ и, тем более, через $\|f\|_{\omega}^{p}$ с $\omega_{f}(t)=(-\ln t)^{-\frac{1}{2 p}}$ (конечно, с некоторой постоянной). А в силу теоремы 4 с той же $\omega_{f}(t)$ справедлива оценка

$$
M(t) \leqslant C(-\ln t)^{-1 / 2}\|f\|_{\omega}^{p}
$$


которая позволяет оценить и второе слагаемое. А именно

$$
\begin{gathered}
\iint_{D}|f(x)| \rho\left(1-|x|^{2}\right)|u|^{p-1} d x \\
\leqslant C\|f\|_{\omega}^{p-1} \int_{1 / 2}^{1}(-\ln r)^{1 / 2-(p-1) /(2 p)}\left(\int_{S_{r}}|f|^{p} d S\right)^{1 / p}\left(1-r^{2}\right) d r \\
\quad+C\|f\|_{L_{p}(|x|<1 / 2)}^{p} \leqslant C\|f\|_{\omega}^{p} .
\end{gathered}
$$

Что дает оценку

$$
\begin{aligned}
\iint_{D}\left(1-|x|^{2}\right) & \left(-\ln \frac{1-|x|^{2}}{2}\right)^{1 / 2} \frac{|\nabla u(x)|^{2}}{u^{2-p}(x)} d x \\
& +\iint_{D}|u|^{p} \frac{1}{\left(1-|x|^{2}\right)\left(-\ln \frac{1-|x|^{2}}{2}\right)^{1 / 2}} d x \leqslant C\|f\|_{\omega}^{p} .
\end{aligned}
$$

с Доказываемое неравенство (1.20) теперь следует из оценки $\|u\|_{C_{n-1, p}(\bar{D})}^{p}$ через левую часть последнего неравенства, которая доказывается так же, как и вложение $W_{2}^{1}(D)$ в $C_{n-1, p}(\bar{D})$ (см. [37], а также [39]).

Справедливость доказываемого утверждения для произвольной функции из $L_{2}(D)$, удовлетворяющей условию (1.19), устанавливается тем же приемом, который был использован в теореме $1^{\prime}$. При доказательстве этой теоремы было показано, что предел решений $u_{k}$ задачи для уравнения с правыми частями $f_{k}$ $\left(f_{k}(x)=f(x)\right.$ при $|x|<1-1 / k$ и $f(x)=0$ для остальных значений $\left.x\right)$ принадлежит $W_{2, \mathrm{loc}}^{1}(D)$, удовлетворяет уравнению и граничному условию. Принадлежность предела пространству $(n-1)$-мерно непрерывных функций и справедливость для него оценки (1.20) немедленно следуют из этой оценки для $u_{k}$, которая уже установлена. Тем самым теорема 2 доказана.

ЗАмечАниЕ 3. В теореме 2 можно ослабить условие на правую часть уравнения. Утверждение останется справедливым, если в качестве $f$ взять такую обобщенную функцию, что

$$
\left|\left\langle f, \rho|v|^{p-1}\right\rangle\right| \leqslant \operatorname{const}\left(\|v\|_{W_{p}^{1}\left(D ; 1-|x|^{2}\right)}+\|v\|_{C_{n-1, p}(\bar{D})}+\|v\|_{L_{p}(|x|<1 / 2)}\right)^{p-1} .
$$

В отличие от замечания 2 это условие на функцию $f$ не содержит выделенного направления и переносится на случай произвольной области.

Приведем еще один вариант теоремы о существовании решения из $C_{n-1, p}(\bar{D})$, который без изменений переносится на случай произвольной области. Отметим, что это утверждение близко к теореме 2, но не следует из нее.

Teорема $3^{\prime}$. Пусть $p \in(1,2)$ u $D=\left\{x \in \mathbf{R}_{n}:|x|<1\right\}$. Тогда для любой $f \in L_{2, \text { lос }}(D)$, удовлетворяющей условию

$$
\int_{D}\left(1-|x|^{2}\right)^{2 p-1}\left(-\ln \left(1-|x|^{2}\right)\right)^{(2 p-1) / 2}|f|^{p} d x<\infty
$$


существует (n-1)-мерно непрерывное решение задачи Дирихле (1.1), (2.1). Это решение единственно и для него справедлива оченка

$$
\begin{aligned}
& \int_{D} r(x)|u|^{p-2}|\nabla u|^{2} d x+\|v\|_{C_{n-1}(\bar{D})}^{p} \\
& \quad \leqslant \operatorname{const} \int_{D}\left(1-|x|^{2}\right)^{2 p-1}\left|\ln \frac{1-|x|^{2}}{2}\right|^{(2 p-1) / 2}|f|^{p} d x
\end{aligned}
$$

в которой постоянная зависит от $n$ и $p$.

ДоКАЗАТЕЛЬСТвО теоремы 3' отличается от доказательства теоремы 2 оценкой правой части неравенства (3.1):

$$
\iint_{D}|f| \eta d x \leqslant\left(\iint_{D} \rho^{2 p-1}|f|^{p} d x\right)^{1 / p}\left(\iint_{D} \frac{|u|^{p}}{\rho} d x\right)^{(p-1) / p} .
$$

Дальнейшие рассуждения дословно повторяют доказательство предыдущего утверждения.

ЗАмЕчАНИЕ 4. Условия (1.19) и (1.20), при которых в теоремах 2 и $3^{\prime}$ доказано существование решения задачи Дирихле (1.1), (2.1), не могут быть точными. При их выполнении в силу теоремы 4 норма решения в $L_{p}\left(S_{r}\right)$ стремится к нулю при $r \rightarrow 1-0$ с фиксированным порядком. Точные условия существования решения неизвестны.

\section{§4. Доказательство теорем 1 и 3 в общем случае}

Доказательство теорем 1 и 3 основывается на покрытии прилегающей к границе части области достаточно малыми подобластями и на оценках решения в этих подобластях. Глобальные оценки устанавливаются их суммированием. Получающийся таким образом результат, естественно, является грубым. Чтобы получить с его помощью нужную для доказательства существования решения, оценку следует “отодвинуться от спектра", рассмотреть уравнение

$$
\sum_{i, j=1}^{n} \frac{\partial}{\partial x_{i}}\left(a_{i j}(x) \frac{\partial u}{\partial x_{j}}\right)-\lambda u=-f(x)
$$

с неотрицательным постоянным коэффициентом $\lambda$; условие эллиптичности (1.4) предполагается выполненным с той же постоянной $\gamma$. Решение задачи Дирихле для этого уравнения мы будем рассматривать в $\S 4$.

Прежде всего опишем покрытие, с которым мы будем работать далее. Возьмем, как и в [37] (см. также [38]), покрытие $\left\{B_{x^{0}}\left(\rho_{0}\right), x^{0} \in \partial Q\right\}$ границы $\partial D$ шарами достаточно малого радиуса $\rho_{0}$. Пересечение области $D$ с каждым из шаров $B_{x^{0}}\left(\rho_{0}\right)$ в местной системе координат $\left(x^{\prime}, x_{n}\right)$ (точка $x^{0}$ является началом координат, а ось $x_{n}$ направлена по нормали $\left.\nu\left(x^{0}\right)\right)$ имеет вид

$$
B_{x^{0}}\left(\rho_{0}\right) \cap D=\left\{\left(x^{\prime}, x_{n}\right) \in B_{x^{0}}\left(\rho_{0}\right): x_{n}>\varphi\left(x^{\prime}\right)\right\}
$$


с некоторой $\varphi=\varphi_{x^{0}} \in C^{1}\left(\mathbf{R}_{n-1}\right)$ (считаем эту функцию определенной на всем $\left.\mathbf{R}_{n-1}\right), \varphi(0)=0, \nabla \varphi(0)=0$ и для всех $x^{\prime}\left|\nabla \varphi\left(x^{\prime}\right)\right|<1 / 2$. Следовательно, для $x \in B_{x^{0}}\left(\rho_{0} / 2\right)$

$$
r(x) \leqslant x_{n}-\varphi\left(x^{\prime}\right)<\frac{4}{3} r(x)
$$

(первое неравенство очевидно). Из покрытия $\left\{B_{x^{0}}\left(\rho_{0} / 2^{5}\right), x_{0} \in \partial Q\right\}$ выберем такое конечное подпокрытие $\left\{B_{x^{k}}\left(\rho_{0} / 2^{5}\right), k=1,2, \ldots, k_{0}\right\}$, что каждая точка пространства принадлежит не более чем $M=M(n)$ шарам $\left\{B_{x^{k}}\left(\rho_{0}\right)\right\}$; шары $B_{x^{k}}\left(\rho_{0} / 2^{7}\right)$ не пересекаются (по поводу возможности такого выбора см. $[46],[47])$. Для каждого $k$ обозначим через $D_{k}$ "криволинейный цилиндр", который в местной системе координат с центром в точке $x^{k}$ имеет вид

$$
D_{k}=\left\{\left(x^{\prime}, x_{n}\right):\left|x^{\prime}\right|<h^{\prime}, \varphi\left(x^{\prime}\right)<x_{n}<\varphi\left(x^{\prime}\right)+h_{n}\right\} ;
$$

число $h^{\prime}$ возьмем равным $\rho_{0} / 2^{4}$, а $h_{n}<\rho_{0} / 2^{4}$ выберем достаточно малым (оно будет зависеть от $n, \gamma, \omega$ и $p)$. Отметим, что $D_{k} \subset B_{x^{0}}\left(\rho_{0} / 2^{3}\right)$ и набор множеств $\bar{D}_{k}$ покрывает $\partial D$

Сначала мы будем рассматривать решение уравнения (4.1) на произвольно взятом элементе выбранного подпокрытия $D_{k}$. Координаты точки будем брать в местной системе координат, а зависимость от номера $k$ будем опускать. Как и в [37], распрямим границу области с помощью отображения $y^{\prime}=x^{\prime}, y_{n}=$ $x_{n}-\varphi\left(x^{\prime}\right)$. В новых координатах уравнение (4.1) имеет такой же вид, его постоянная эллиптичности оценивается снизу (с абсолютной положительной постоянной) через постоянную эллиптичности $\gamma$, а коэффициенты уравнения в новых координатах удовлетворяют условию (1.12), в котором функцию $\omega(t)$ следует заменить на $C(n, \gamma) \omega(\sqrt{2} t), C(n, \gamma) \geqslant 1$ (см. [37]); далее эту новую функцию будем также обозначать через $\omega$. Новые координаты, коэффициенты уравнения в них, постоянную эллиптичности этого уравнения и решение будем обозначать теми же буквами $x=\left(x^{\prime}, x_{n}\right), a_{i j}, \gamma$ и $u$.

При интегрировании по частям нам понадобится специальное сглаживание коэффициентов. Как и в [37], рассмотрим функции $a_{i j}^{0}, i, j=1, \ldots, n$, определенные равенствами

$$
\begin{aligned}
& a_{i j}^{0}(x)=a_{i j}(x) \quad \text { при } \quad i<n, j<n, \\
& \begin{aligned}
a_{i n}^{0}(x)= & a_{n i}^{0}(x)=a_{n i}^{0}\left(x^{\prime}, x_{n}\right)=\frac{1}{\operatorname{mes}_{n-1}\left\{\xi \in \mathbf{R}_{n-1}:|\xi|<x_{n}\right\}} \\
& \times \int_{\left\{\xi \in \mathbf{R}_{n-1}:\left|\xi-x^{\prime}\right|<x_{n}\right\}} a_{i n}(\xi, 0) d \xi \quad \text { при } \quad i=1, \ldots, n-1,
\end{aligned} \\
& a_{n n}^{0}(x)=a_{n n}\left(x^{\prime}, 0\right) ;
\end{aligned}
$$

матрицу коэффициентов $a_{i j}$ будем обозначать через $A$, а сглаженную матрицу $\left(a_{i j}^{0}\right)$ - через $A_{0}$. Из условия (1.12) следует, что

$$
\begin{aligned}
& \left|a_{i n}^{0}(x)-a_{i n}(x)\right| \leqslant \omega\left(\sqrt{2} x_{n}\right), \quad i=1,2, \ldots, n, \\
& \left|\frac{\partial a_{i n}^{0}(x)}{\partial x_{i}}\right| \leqslant \frac{n-1}{x_{n}} \omega\left(2 x_{n}\right), \quad i=1,2, \ldots, n-1 .
\end{aligned}
$$


Договоримся и функцию $n \omega(2 t)$ также обозначать через $\omega(t)$. Тогда

$$
\left[\sum_{i=1}^{n}\left(a_{i n}(x)-a_{i n}^{0}(x)\right)^{2}\right]^{1 / 2} \leqslant \omega\left(x_{n}\right), \quad\left|\frac{\partial a_{i n}^{0}(x)}{\partial x_{i}}\right| \leqslant \frac{\omega\left(x_{n}\right)}{x_{n}}, \quad i=1, \ldots, n-1 .
$$

Обозначим через П цилиндр

$$
\left\{x=\left(x^{\prime}, x_{n}\right):\left|x^{\prime}\right|<h^{\prime}, 0<x_{n}<h_{n}\right\}
$$

с $h^{\prime}=\rho / 2^{4},-$ образ $D_{k}$ при взятом распрямляющем отображении, а

$$
c \Pi=\left\{x=\left(x^{\prime}, x_{n}\right):\left|x^{\prime}\right|<c h^{\prime}, 0<x_{n}<c h_{n}\right\}, \quad c>0 ;
$$

индекс $k$ у обозначения этого цилиндра мы опускаем. Заметим, что прообразы всех таких цилиндров $\frac{1}{2} \bar{\Pi}$ покрывают границу $\partial D$ рассматриваемой области $D$.

4.1. Доказательство теоремы 1. Как и в доказательстве теоремы $1^{\prime}$ в $\S 2$, рассмотрим сначала случай, когда правая часть рассматриваемого уравнения (4.1) принадлежит пространству $L_{2}(D)$, а ее носитель лежит в области $D$. Тогда обобщенное решение $u$ задачи Дирихле (4.1), (2.1) принадлежит пространству $\stackrel{\circ}{W}_{2}^{1}(D)$. Далее мы будем рассматривать это решение (точнее, суперпозицию этой функции и отображения, обратного к распрямляющему) в цилиндре 2П. При этом, как мы договорились выше, будем использовать те же обозначения координат (в местной системе) и коэффициентов уравнения и решения.

Возьмем произвольное число $t \in\left(0, h_{n}\right)$ и обозначим

$$
\rho=\rho(x, t)=\min \left\{x_{n}, t\right\} .
$$

Кроме того, пусть $\psi^{\prime}\left(x^{\prime}\right), x^{\prime} \in \mathbf{R}_{n-1}$ и $\psi_{n}\left(x_{n}\right), x_{n} \geqslant 0$ - бесконечно дифференцируемые срезающие функции, принимающие значения в отрезке $[0,1]$ и удовлетворяющие условиям

$$
\begin{array}{rlll}
\psi^{\prime}\left(x^{\prime}\right)=1 & \text { при } & \left|x^{\prime}\right| \leqslant h^{\prime}, \\
\psi^{\prime}\left(x^{\prime}\right)=0 & \text { при } & \left|x^{\prime}\right| \geqslant 2 h^{\prime}, \\
\psi_{n}\left(x_{n}\right)=1 & \text { при } & x_{n} \in\left[0, h_{n}\right], \\
\psi_{n}\left(x_{n}\right)=0 & \text { при } & x_{n} \geqslant 2 h_{n} ; \\
\left|\nabla \psi^{\prime}\left(x^{\prime}\right)\right| \leqslant \frac{C}{h^{\prime}}, & \left|\frac{\partial \psi_{n}}{\partial x_{n}}\right| \leqslant \frac{C}{h_{n}}
\end{array}
$$

с абсолютной постоянной $C$. Произведение $\psi^{\prime} \psi_{n}$ будем обозначать через $\psi$.

Возьмем произвольное положительное число $\delta$ и подставим в определяющее решение интегральное тождество пробную функцию

$$
\eta=\eta_{\delta}(x)=\rho(x, t)\left(u^{+}(x)+\delta\right)^{p-2} u^{+}(x)
$$

здесь, как и ранее, $u^{+}=1 / 2(|u|+u)$, а $u^{-}=1 / 2(|u|-u)$. Очевидно, что функция $\eta$ принадлежит $\stackrel{\circ}{2}_{2}^{1}(D)$; напомним, что мы рассматриваем случай $p \in(1,2)$. 
Получим

$$
\begin{aligned}
& \iint_{2 \Pi} f(x) \eta(x) d x=\iint_{2 \Pi}(\nabla u, A \nabla \eta) d x \\
& =\iint_{2 \Pi} \psi \rho\left(\nabla u^{+}, A \nabla u^{+}\right)\left[\frac{1}{\left(u^{+}+\delta\right)^{2-p}}+\frac{(p-2) u^{+}}{\left(u^{+}+\delta\right)^{3-p}}\right] d x \\
& \quad+\lambda \iint_{2 \Pi} \psi \rho\left(u^{+}+\delta\right)^{p-2} u^{+^{2}} d x+\iint_{2 \Pi_{t}} \psi^{\prime}\left(u^{+}+\delta\right)^{p-2} u^{+}\left(e_{n}, A \nabla u\right) d x \\
& \quad+\iint_{2 \Pi \backslash \Pi} \rho\left(u^{+}+\delta\right)^{p-2} u^{+}(\nabla \psi, A \nabla u) d x
\end{aligned}
$$

здесь и далее $2 \Pi_{t}=\left\{x \in 2 \Pi: x_{n}<t\right\}-$ множество тех точек 2П, в которых $\rho(x, t)=x_{n}$, а $e_{n}-$ направляющий вектор оси $x_{n}$.

В силу (1.4) первое слагаемое правой части (4.4) оценивается снизу величиной

$$
\gamma(p-1) \iint_{2 \Pi} \psi \rho \frac{\left|\nabla u^{+}\right|^{2}}{\left(u^{+}+\delta\right)^{2-p}} .
$$

Остальные слагаемые и левая часть имеют пределы при $\delta \rightarrow+0$; напомним, что $u \in \stackrel{\circ}{W}_{2}^{1}(D)$. Как и в п. 2.1, отсюда получаем, что и интеграл в последней формуле стремится к

$$
\iint_{2 \Pi} \psi \rho \frac{\left|\nabla u^{+}\right|^{2}}{u^{+^{2-p}}} d x .
$$

Предел третьего слагаемого проинтегрируем по частям, предварительно сгладив коэффициенты:

$$
\begin{aligned}
\int_{2 \Pi^{\prime}} & \psi^{\prime}\left(x^{\prime}\right) \frac{a_{n n}\left(x^{\prime}, 0\right)}{p} u^{+p}\left(x^{\prime}, t\right) d x^{\prime}+\iint_{2 \Pi_{t}} \psi^{\prime} u^{+p-1}\left(e_{n},\left(A-A_{0}\right) \nabla u\right) d x \\
+ & \frac{1}{p} \iint_{2 \Pi_{t}} u^{+p} \sum_{k=1}^{n-1}\left[a_{n k}^{0} \frac{\partial \psi^{\prime}}{\partial x_{k}}+\psi^{\prime} \frac{\partial a_{n k}^{0}}{\partial x_{k}}\right], \quad \Pi^{\prime}=\left\{x^{\prime} \in \mathbf{R}_{n-1}:\left|x^{\prime}\right|<h^{\prime}\right\} .
\end{aligned}
$$

Проведя аналогичные рассуждения для $u^{-}$и сложив полученные неравенства, с помощью оценок (4.3) и выбора достаточно малого $h_{n}=h_{n}(n, \gamma, \omega, p)$ получим

$$
\begin{aligned}
& \iint_{2 \Pi} \psi \rho \\
& \quad+\frac{|\nabla u|^{2}}{|u|^{2-p}} d x+\lambda \iint_{2 \Pi} \psi \rho|u|^{p} d x+\max _{0 \leqslant \tau \leqslant t} \int_{2 \Pi^{\prime}} \psi^{\prime}|u|^{p} d x \\
& \leqslant \varepsilon \iint_{2 \Pi \backslash \Pi} \rho \frac{|\nabla u|^{2}}{|u|^{2-p}} d x+\frac{C}{\varepsilon}\left(\iint_{\left\{x \in 2 \Pi_{t}:\left|x^{\prime}\right|>h^{\prime}\right\}}|u|^{p} d x+\iint_{\left\{x \in 2 \Pi: x_{n}>t\right\}}|u|^{p} d x\right) \\
& \quad+C \int_{0}^{2 h_{n}} \rho\left\|f\left(\cdot, x_{n}\right)\right\|_{L_{p}\left(2 \Pi^{\prime}\right)} d x_{n} ;
\end{aligned}
$$

здесь $\varepsilon$ - достаточно малое число (зависящее, как и все постоянные, только от $n, \gamma, \omega$ и $p)$. Делая обратную замену переменных и суммируя полученные 
оценки с $t=h_{n}$ по всем элементам покрытия, имеем неравенство

$$
\begin{aligned}
& \iint_{\{x \in D: r(x)<\delta\}} r(x) \frac{|\nabla u|^{2}}{|u|^{2-p}} d x+\lambda \iint_{\{x \in D: r(x)<\delta\}} r(x)|u|^{p} d x \\
& \quad+\sup _{0<t<\delta} \frac{1}{t} \iint_{\{x \in D: r(x)<t\}}|u|^{p} d x \\
& \leqslant C \iint_{\left\{\delta<r(x)<3 h_{n}\right\}}\left(\frac{|\nabla u|^{2}}{|u|^{2-p}}+|u|^{p}\right) d x+C \iint_{D} \frac{r(x)^{2 p-1}}{\left[\omega_{f}(r(x))\right]^{p-1}}|f(x)|^{p} d x,
\end{aligned}
$$

в котором $\delta=\delta(n, \gamma, \omega, p)$ - такое положительное число, что приграничная полоса $\{x \in D: r(x)<\delta\}$ лежит в объединении $D_{k}$.

Возьмем теперь подобласть $D_{0}$,

$$
\{x \in D: r(x)>\delta\} \subset D_{0} \subset\left\{r(x)>\frac{\delta}{2}\right\}
$$

и стандартным образом (см., например, [38]) оценим сумму интегралов

$$
\iint_{\{x \in D: r(x)>\delta\}} \frac{|\nabla u|^{2}}{|u|^{2-p}} d x+\lambda \iint_{\{x \in D: r(x)>\delta\}}|u|^{p} d x
$$

через

$$
\operatorname{const}\left(\iint_{\{r(x)>\delta / 2\}}|u|^{p} d x+\iint_{\{r(x)>\delta / 2\}}|f(x)|^{p} d x\right) .
$$

Отсюда немедленно следует, что

$$
\begin{aligned}
& \iint_{D} r(x) \frac{|\nabla u|^{2}}{|u|^{2-p}} d x+\lambda \iint_{D} r(x)|u|^{p} d x+\sup _{0<t<\delta} \frac{1}{t} \iint_{\{x \in D: r(x)<t\}}|u|^{p} d x \\
& \quad \leqslant \operatorname{const}\left(\iint_{\{x \in D: r(x)>\delta / 2\}}|u|^{p} d x+\iint_{D} \frac{r(x)^{2 p-1}}{\left[\omega_{f}(r(x))\right]^{p-1}}|f(x)|^{p} d x\right),
\end{aligned}
$$

здесь постоянная зависит еще и от диаметра области $D$.

Таким образом, справедливо следующее вспомогательное утверждение.

Лемма 4. Существует такое число $\lambda_{0}>0$, что решение задачи Дирихле (4.1), (2.1) с $\lambda=\lambda_{0} u f \in L_{2}(D)$, supp $f \subset D$, удовлетворяет оченке (1.16).

Докажем теперь справедливость оценки (1.16) для интересующего нас случая $\lambda=0$. Наряду с решением $u$ рассмотрим решение $w$ задачи с $\lambda=\lambda_{0}$ из леммы 4

$$
\sum_{i, j=1}^{n} \frac{\partial}{\partial x_{i}}\left(a_{i j}(x) \frac{\partial w}{\partial x_{j}}\right)-\lambda_{0} w=f, \quad x \in D,\left.\quad w\right|_{\partial Q}=0 .
$$

По лемме 4 это решение удовлетворяет оценке (1.16). В частности,

$$
\int_{D}|w(x)|^{p} d x \leqslant C \int_{D} \frac{r(x)^{2 p-1}}{\left[\omega_{f}(r(x))\right]^{p-1}}|f(x)|^{p} d x .
$$

4 Математический сборник, т. 206, вып. 10 
Разность $z=u-w$ является решением задачи (1.3), (2.1) с правой частью $-\lambda_{0} w$. Но тогда в силу леммы 2 из работы [38] и (4.6) справедлива оценка

$$
\|u\|_{L_{p}(D)} \leqslant\|w\|_{L_{p}(D)}+\|z\|_{L_{p}(D)} \leqslant C\left(\int_{D} \frac{r(x)^{2 p-1}}{\left[\omega_{f}(r(x))\right]^{p-1}}|f(x)|^{p} d x\right)^{1 / p},
$$

подставляя которую в (4.5), немедленно получаем требуемое неравенство (1.16).

Окончание доказательства теоремы 1 практически дословно повторяет соответствующие рассуждения из доказательства теоремы $1^{\prime}$ в $\S 2$. Следует только кратко остановиться на проверке выполнения граничного условия. Нужно доказать, что для любой точки $x^{0} \in \partial D$ найдется такая окрестность $V_{x^{0}} \subset \partial D$, что выполняется (1.11). Для этого возьмем произвольную точку $x^{0} \in \partial D$ и выбор конечного подпокрытия построенного в начале этого параграфа покрытия следует начать с окрестности точки $x^{0}$. В силу теоремы единственности решение не зависит от выбора подпокрытия. Таким образом, теорема 1 доказана.

4.2. Доказательство теоремы 3. Так как из условий теоремы 3 следует выполнение условия теоремы 1 , то существование решения из $W_{2, \text { loc }}^{1}(D)$ установлено; далее мы будем пользоваться построениями и результатами, установленными при доказательстве теоремы 1, сохраняя те же обозначения. Как и при доказательствах предыдущих теорем, достаточно рассмотреть финитную правую часть (supp $f \subset D)$; справедливость утверждения в общем случае устанавливается тем же предельным переходом.

Прежде всего рассмотрим решение $u$ в области $D_{0}, \bar{D}_{0} \subset D$. Так как $u \in W_{2}^{1}\left(D_{0}\right)$, то оно принадлежит $C_{n-1,2}\left(\bar{D}_{0}\right)$, а следовательно, и $C_{n-1, p}\left(\bar{D}_{0}\right)$; напомним, что $p<2$. Кроме того, функция $v=|u|^{p / 2} \in W_{2}^{1}\left(D_{0}\right)$ и

$$
\begin{aligned}
\|v\|_{W_{2}^{1}\left(D_{0}\right)}^{2} & =\iint_{D_{0}}|u|^{p} d x+\frac{p^{2}}{4} \iint_{D_{0}}|u|^{p-2}|\nabla u|^{2} d x \\
& \leqslant \text { const } \int_{D}(r(x))^{2 p-1}|\ln (r(x))|^{(2 p-1) / 2}|f|^{p} d x .
\end{aligned}
$$

Откуда

$$
\begin{aligned}
\|u\|_{C_{n-1, p}\left(\bar{D}_{0}\right)} & =\|v\|_{C_{n-1,2}\left(\bar{D}_{0}\right)} \leqslant C\|v\|_{W_{2}^{1}\left(D_{0}\right)} \\
& \leqslant C \int_{D}(r(x))^{2 p-1}|\ln (r(x))|^{(2 p-1) / 2}|f|^{p} d x .
\end{aligned}
$$

Теперь нам достаточно (см. лемму 2) установить соответствующую оценку на произвольном элементе покрытия границы (принадлежность решения пространству $C_{n-1, p}$ следует из вложения в него $W_{2}^{1}$. А для этого в силу оценок (4.2) достаточно получить эти результаты в цилиндре П. Эту ситуацию мы и будем рассматривать далее.

Возьмем, как и в доказательстве теоремы 2 , вес $\rho(t)=t(-\ln t)^{1 / 2}$ и подставим в интегральное тождество пробную функцию

$$
\eta=\eta_{\delta}=\psi^{\prime 2} \psi_{n} \rho\left(u^{+}+\delta\right)^{p-2} u^{+}, \quad \delta>0 .
$$


Переходя в полученном равенстве к пределу при $\delta \rightarrow+0$ и складывая полученный результат с аналогичным равенством для $u^{-}$, получаем

$$
\begin{aligned}
& \iint_{2 \Pi} f \psi^{\prime 2} \psi_{n} \rho|u|^{p-1} d x \\
& =(p-1) \iint_{2 \Pi}{\psi^{\prime}}^{2} \psi_{n} \rho \frac{(\nabla u, A \nabla u)}{|u|^{2-p}} d x+\iint_{2 \Pi} \psi^{\prime 2} \psi_{n} \frac{d \rho}{d x_{n}}|u|^{p-1}\left(e_{n}, A \nabla|u|\right) d x \\
& \quad+\iint_{2 \Pi} \psi^{\prime 2} \frac{d \psi_{n}}{d x_{n}} \rho|u|^{p-1}\left(e_{n}, A \nabla|u|\right) d x+\iint_{2 \Pi} \psi_{n} \rho|u|^{p-1}\left(\nabla \psi^{\prime 2}, A \nabla|u|\right) .
\end{aligned}
$$

Оценивая аналогично тому, как это делалось при доказательстве теорем 1 и 2 , слагаемые в последнем неравенстве, имеем оценку

$$
\begin{aligned}
& \iint_{2 \Pi} \psi_{n} \rho \frac{\left|\nabla\left[\psi^{\prime}|u|\right]\right|^{2}}{|u|^{2-p}} d x+\iint_{2 \Pi} \psi_{n}\left[\psi^{\prime}|u|^{p / 2}\right]^{2} \frac{1}{\rho} d x \\
& \quad \leqslant C\|u\|_{L_{p}(2 \Pi)}^{p}+C\left(\iint_{2 \Pi} x_{n}^{2 p-1}\left(-\ln x_{n}\right)^{(2 p-1) / 2}|f|^{p} d x\right)\left(\iint_{2 \Pi}\left[\psi^{\prime}|u|^{p / 2}\right]^{2} \frac{1}{\rho} d x\right) .
\end{aligned}
$$

Доказываемое утверждение, как и в доказательстве теоремы 2, следует из оценки $\|u\|_{C_{n-1, p}(\bar{\Pi})}^{p}$ через левую часть последнего неравенства.

ЗАмечАниЕ 5. В теореме 4, как и в теореме 2, можно ослабить условие на правую часть уравнения. Утверждение останется справедливым, если в качестве $f$ взять такую обобщенную функцию, что

$$
\left|\left\langle f, \rho|v|^{p-1}\right\rangle\right| \leqslant \operatorname{const}\left(\|v\|_{W_{p}^{1}(D ; r(x))}+\|v\|_{C_{n-1, p}(\bar{D})}\right)^{p-1}
$$

для всех $v \in W_{p}^{1}(D) \cap C_{n-1, p}(\bar{D})$. В частности, теорема верна для таких $f$, что $f / \varrho$ является линейным ограниченным функционалом на $C_{n-1, p /(p-1)}(\bar{D})$.

\section{Список литературы}

[1] A. Liapounoff, "Sur certaines questions qui se rattachent au problème de Dirichlet", J. Math. Pures Appl. (5), 4 (1898), 241-311.

[2] H. Poincaré, "La méthode de Neumann et le probléme de Dirichlet", Acta Math., 20:1 (1896), 59-142.

[3] H. Poincaré, "Sur les equations aux dérivées partielles de la physique mathématique", Amer. J. Math., 12:3 (1890), 211-294.

[4] A. Korn, Lehrbuch der Potentialtheorie. Allgemeine Theorie des Potentials und der Potentialfunctionen im Raume, Ferd. Dümmler, Berlin, 1899, xiv+417 pp.

[5] W. Stekloff, "Sur les problèmes fondamentaux de la physique mathématique", C. $R$. Acad. Sci. Paris, 128 (1899), 588-591.

[6] W. Stekloff, "Les méthodes générales pour résoudre les problèmes fondamentaux de la physique mathématique", Ann. Fac. Sci. Toulouse Sci. Math. Sci. Phys. (2), 2:2 (1900), 207-272.

[7] S. Zaremba, "Sur la théorie de l'équation de Laplace et les méthodes de Neumann et de Robin", Bull. Acad. Sci. Cracovie, 41 (1901), 171-189.

[8] В. А. Стеклов, Основные задачи математической физики, Наука, М., 1983, 432 с. 
[9] O. Hölder, Beiträge zur Potentialtheorie, Inaugural-Diss., Univ. Tübingen, 1882, $78 \mathrm{pp}$.

[10] Д. Гилбарг, Н. Трудингер, Эллиптические дифференциалъные уравнения с частными производными второго порядка, Наука, М., 1989, 464 с.; пер. с англ.: D. Gilbarg, N.S. Trudinger, Elliptic partial differential equations of second order, 2nd ed., Grundlehren Math. Wiss., 224, Springer-Verlag, Berlin, 1983, xiii+513 pp.

[11] A. Korn, Über Minimalflächen, deren Randkurven wenig von ebenen Kurven abweichen, Königl. Akad. Wiss., Berlin, 1909, 37 pp.

[12] E. Hopf, "Elementare Bemerkungen über die Lösungen partieller Differentialgleichungen zweiter Ordnung vom elliptischen Typus", Sitzungsber. Preuß. Akad. Wiss., Phys.-Math. Kl., 19 (1927), 147-152 pp.

[13] A. Korn, "Zwei Anwendungen der Methode der sukzessiven Annäherungen", Schwarz Festschrift, Julius Springer, Berlin, 1914, 215-229 pp.

[14] G. Giraud, "Sur le problème de Dirichlet généralisé (deuxième mémoire)", Ann. Sci. École Norm. Sup. (3), 46 (1929), 131-245.

[15] G. Giraud, "Sur certains problèmes non linéaires de Neumann et sur certains problèmes non linéaires mixtes", Ann. Sci. École Norm. Sup. (3), 49 (1932), 1-104.

[16] G. Giraud, "Sur certains problèmes non linéaires de Neumann et sur certains non linéaires mixtes", Ann. Sci. École Norm. Sup., 49:3 (1932), 245-309.

[17] J. Schauder, "Über lineare elliptische Differentialgleichungen zweiter Ordnung", Math. Z., 38:1 (1934), 257-282.

[18] J. Schauder, "Numerische Abschätzungen in elliptischen linearen Differentialgleichungen", Studia Math., 5 (1934), 34-42.

[19] E. Hopf, "Über den funkitonalen, insbesondere den analytischen Charakter der Lösungen elliptischer Differentialgleichungen zweiter Ordnung", Math. Z., 34:1 (1932), $194-233$.

[20] H. Lebesgue, "Sur le problème de Dirichlet", Rend. Circ. Mat. Palermo, 24 (1907), 371-402.

[21] O. Perron, "Eine neue Behandlung der ersten Randwertaufgabe für $\Delta u=0$ ", Math. Z., 18:1 (1923), 42-54.

[22] И. Г. Петровский, Лекиии об уравнениях с частными производными, 2-е изд., Гостехиздат, М., 1953, 360 с.; англ. пер. 1-го изд.: I. G. Petrovsky, Lectures on partial differential equations, Interscience Publishers, New York-London, 1954, x+245 pp.

[23] N. Wiener, "The Dirichlet problem", J. Math. Phys. Mass. Inst. Tech., 3 (1924), $127-146$.

[24] М. В. Келдыш, "О разрешимости и устойчивости задачи Дирихле”, УМН, 1941, № 8, 171-231; англ. пер.: M. V. Keldysh, "On the solvability and stability of the Dirichlet problem", Amer. Math. Soc. Transl. Ser. 2, 51, Amer. Math. Soc., Providence, R.I., 1966, 1-73.

[25] О. А. Олейник, "О задаче Дирихле для уравнений эллиптического типа", Матем. сб., 24(66):1 (1949), 3-14.

[26] С. Л. Соболев, "О некоторых оценках, относящихся к семействам функций, имеющих производные, интегрируемые с квадратом”, Докл. АН СCCP, 1:7 (1936), 267-270.

[27] С.Л. Соболев, "Méthode nouvelle à résoudre le probléme de Cauchy pour les équations linéaires hyperboliques normales", Матем. сб., 1(43):1 (1936), 39-72.

[28] С. Л. Соболев, “Об одной теореме функционального анализа”, Матем. сб., 4(46):3 (1938), 471-497; англ. пер.: S. L. Sobolev, "On a theorem of functional analysis", Amer. Math. Soc. Transl. Ser. 2, 2(34), Amer. Math. Soc., Providence, R.I., 1963, 39-68. 
[29] С. Л. Соболев, Некоторые применения функиионального анализа в математической физике, 2-е изд., Изд-во СО АН СССР, Новосибирск, 1962, 255 с.; англ. пер. 1-го изд.: S. L. Sobolev, Applications of functional analysis in mathematical physics, Transl. Math. Monogr., 7, Amer. Math. Soc., Providence, R.I., 1963, vii+239 pp.

[30] D. Hilbert, "Über das Dirichlet'sche Prinzip", Jahresber. Deutsch. Math.-Ver., 8:1 (1900), 184-188.

[31] H. Lebesgue, "Sur le problème de Dirichlet", Rend. Circ. Mat. Palermo, 24 (1907), 371-402.

[32] В. С. Владимиров, Обобщенные функиии в математической физике, 2-е изд., Наука, M., 1979, 319 с.; англ. пер.: V.S. Vladimirov, Generalized functions in mathematical physics, Mir, Moscow, 1979, xii+362 pp.

[33] О.А. Ладыженская, Н. Н. Уральцева, Линейные и квазилинейные уравнения эллиптического типа, 2-е изд., Наука, М., 1973, 576 с.; англ. пер. 1-го изд.: O.A. Ladyzhenskaya, N.N. Ural'tseva, Linear and quasilinear elliptic equations, Academic Press, New York-London, 1968, xviii+495 pp.

[34] И. Нечас, "О решениях эллиптических дифференциальных уравнений в частных производных второго порядка с неограниченным интегралом Дирихле", Чехослов. матем. журн., 10:2 (1960), 283-298.

[35] В.П. Михайлов, "О задаче Дирихле для эллиптического уравнения второго порядка", Дифферени. уравнения, 12:10 (1976), 1877-1891; англ. пер.: V.P. Mikha1lov, "Dirichlet's problem for a second-order elliptic equation", Differential Equations, 12(1976):10 (1977), 1320-1329.

[36] И. М. Петрушко, “О граничных значениях в $\mathscr{L}_{p}, p>1$, решений эллиптических уравнений в областях с ляпуновской границей”, Матем. сб., 120(162):4 (1983), 569-588; англ. пер.: I. M. Petrushko, "On boundary values in $\mathscr{L}_{p}, p>1$, of solutions of elliptic equations in domains with a Lyapunov boundary", Math. USSR-Sb., 48:2 (1984), 565-585.

[37] А. К. Гущин, "О задаче Дирихле для эллиптического уравнения второго порядка", Матем. сб., 137(179):1(9) (1988), 19-64; англ. пер.: A. K. Gushchin, "On the Dirichlet problem for a second-order elliptic equation", Math. USSR-Sb., 65:1 (1990), $19-66$.

[38] А. К. Гущин, "О задаче Дирихле для эллиптического уравнения второго порядка с граничной функцией из $L_{p}$ ", Матем. сб., 203:1 (2012), 3-30; англ. пер.: A. K. Gushchin, "The Dirichlet problem for a second-order elliptic equation with an $L_{p}$ boundary function", Sb. Math., 203:1 (2012), 1-27.

[39] А.К. Гущин, В.П. Михайлов, "О существовании граничных значений решений эллиптических уравнений”, Матем. сб., 182:6 (1991), 787-810; англ. пер.: A. K. Gushchin, V.P. Mikhailov, "On the existence of boundary values of solutions of an elliptic equation", Math. USSR-Sb., 73:1 (1992), 171-194.

[40] L. Carleson, "An interpolation problem for bounded analytic functions", Amer. J. Math., 80 (1958), 921-930.

[41] L. Carleson, "Interpolations by bounded analytic functions and the corona problem", Ann. of Math. (2), 76:3 (1962), 547-559.

[42] L. Hörmander, " $L^{p}$-estimates for (pluri-) subharmonic functions", Math. Scand., 20 (1967), 65-78.

[43] А.К. Гущин, " $L_{p}$-оценки решения задачи Дирихле для эллиптического уравнения второго порядка", ТМФ, 174:2 (2013), 243-255; англ. пер.: A. K. Gushchin, " $L_{p}$-estimates for solutions of second-order elliptic equation Dirichlet problem", Theoret. and Math. Phys., 174:2 (2013), 209-219.

[44] В.Ж. Думанян, "О разрешимости задачи Дирихле для общего эллиптического уравнения второго порядка", Матем. сб., 202:7 (2011), 75-94; англ. пер.: 
V.Zh. Dumanyan, "Solvability of the Dirichlet problem for a general second-order elliptic equation", Sb. Math., 202:7 (2011), 1001-1020.

[45] В. Ж. Думанян, "О разрешимости задачи Дирихле для эллиптического уравнения второго порядка”, ТМФ, 180:2 (2014), 189-205; англ. пер.: V. Zh. Dumanyan, "Solvability of the Dirichlet problem for second-order elliptic equations", Theoret. and Math. Phys., 180:2 (2014), 917-931.

[46] A. P. Morse, "A theory of covering and differentiation", Trans. Amer. Math. Soc., 55 (1944), 205-235.

[47] В.Г. Мазья, "О вырождающейся задаче с косой производной", Матем. сб., 87(129):3 (1972), 417-454; англ. пер.: V. G. Maz'ya, "On a degenerating problem with directional derivative", Math. USSR-Sb., 16:3 (1972), 429-469.

\section{Анатолий Константинович Гущин} (Anatoliy K. Gushchin)

Поступила в редакцию

Математический институт им. В.А. Стеклова

18.06 .2015

Российской академии наук, г. Москва

E-mail: akg@mi.ras.ru 